Fourier transform of $G_{1}(\vec{r})$. The correct Fourier transform of $G_{1}(\overrightarrow{\mathrm{r}})$ is $\hat{G}_{1}(\overrightarrow{\mathrm{q}})$ [Eq. (4a)].

${ }^{13}$ M. P. Schulhof, P. Heller, R. Nathans, and A. Linz, Phys. Rev. B 1 , 2304 (1970).

${ }^{14}$ A. Tucciarone, H. Y. Lau, L. M. Corliss, A. Delapalme, and J. M. Hastings, Phys. Rev. B $\underline{4}, 3206$ (1971).

${ }^{15}$ C. C. Lai and S. H. Chen, Phys. Letters $41 \mathrm{~A}, 259$ (1972).

${ }^{16}$ D. S. Ritchie and M. E. Fisher [Phys. Rev. B $\underline{5}$, $2668(1972)]$ have found that $\hat{G}_{3}$ also accurately describes the Heisenberg model.

${ }^{17}$ For small $\eta$ the ratio $C(q \xi)$ is approximately linear in $\eta$; therefore, our results, obtained for $\eta=0.05$ and $\eta$ $=0.10$, can be interpolated in order to analyze linewidth data for other values of $\eta$.

${ }^{18}$ In the limit $q \xi \gg 1$ we find $\Gamma_{q s}\left(\hat{G}_{3}, \eta, q \xi\right)=\Gamma_{q s}\left(\hat{G}_{2}, \eta, q \xi\right)$, for any value of the parameter $\phi$ in $\hat{G}_{3}$.

${ }^{19}$ B. Chu, S. P. Lee, and W. Tscharnuter, Phys. Rev. A 7,353 (1973).

\title{
Exact Photocount Statistics: Lasers near Threshold
}

\author{
M. Lax* and M. Zwanziger ${ }^{\dagger \neq}$ \\ Bell Telephone Laboratories, Murray Hill, New Jersey 07974
}

(Received 8 November 1971; revised manuscript received 31 July 1972)

\begin{abstract}
A previously constructed laser model with quantum (noncommuting) noise sources was shown to lead near threshold to a quantum rotating-wave Van der Pol oscillator. A full dynamical correspondence between quantum and classical random processes allows one to compute the average of any time-ordered, normal-ordered operator function by averaging the associated function of classical random variables. Numerical calculations for the associated classical Van der Pol oscillator of the steady-state distribution, the total intensity fluctuations, and the linewidth versus operating point were amply confirmed experimentally. Measurements (and calculations) of higher than two-time correlations were sparse and contradictory. Photocount distributions, at times short compared to the intensity correlation time, confirm only the steady state of the laser. Photocount distributions at intermediate and longer times are difficult to compute because they involve multitime correlations of high $(\infty)$ order. By providing an exact solution for photocount distributions and their moments for all times, we expected to stimulate measurements near threshold which would provide an adequate test of the Van der Pol laser model. Comparison of the results reported here with recent photocount experiments of Meltzer, Davis, and Mandel and of Jakeman, Oliver, and Pike provides gratifying agreement and confirmation of our statistical understanding of laser fluctuations near threshold.
\end{abstract}

\section{INTRODUCTION}

The present paper provides an "exact" (i. e., with no stochastic approximations) solution of a long-standing problem in laser statistics: the probability $p(m, T)$ of observing $m$ photocounts in a time $T$ produced in a photodetector by a laser operating in the vicinity of threshold for all times $T$, short, comparable to, or long, compared to the laser-intensity correlation time $T_{c}$. Well above threshold, laser fluctuations are negligible, and the photocount distribution reduces to a Poisson distribution. Well below threshold, the statistics are Gaussian, and the problem reduces to a wellknown but nontrivial problem on fluctuations of time-integrated intensities of a Gaussian variable for which exact numerical solutions have been given. ${ }^{1-3}$ The region near threshold holds a special interest because the onset of lasing is a phase transition, ${ }^{4,5}$ and the region near threshold is equivalent to the critical region near the transition temperature in a second-order phase transition.

Our solution is exact in that no quantum-mechanical or stochastic approximations are made in treating the rotating-wave Van der Pol (RWVP) model of a laser (described more fully in Sec. IV). Of course, many key approximations were made in arriving at this model: (i) The atom-field system was treated as a Markoffian system with noncommuting noise sources ${ }^{6,7-13}$; (ii) the atomic variables were adiabatically eliminated by assuming that they responded to the instantaneous field variables ${ }^{14}$; (iii) restoring forces higher than quadratic in the intensity were then neglected near threshold with a fractional error ${ }^{15}$ of the order of $1 / n^{\text {th }}$ where $n^{\text {th }} \sim 10^{4}$ is the number of photons at threshold. The Markoffian approximation assumes that the duration of a collision with a reservoir atom $\left(\sim 10^{-12} \mathrm{sec}\right)$ is short compared to the mean time $\left(\Gamma^{-1} \sim 10^{-8} \mathrm{sec}\right)$ between collisions -an excellent approximation used in the Boltzmann description of gases. The adiabatic approximation appears to assume that atomic decay rates $\left(\Gamma \sim 10^{8} /\right.$ sec) are fast compared to photon decay rates $\left(\gamma \sim 10^{7} / \mathrm{sec}\right)$, but actually involves the much weaker approximation that all of these rates are fast compared to the intensity relaxation rate near threshold, ${ }^{16} \sim \gamma / n^{\text {th }}$. These remarks support our gener- 


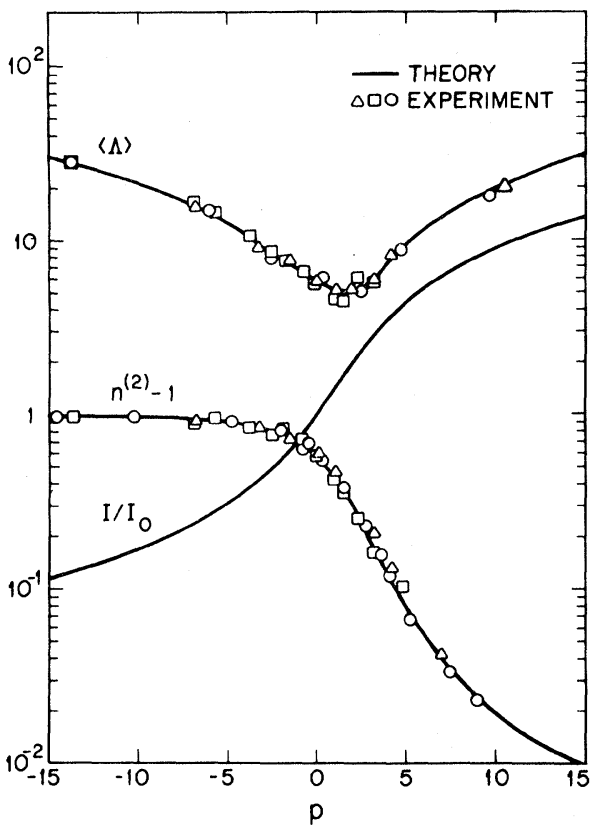

FIG. 1. Experimental verification of the Van der Pol model for lasers operating near threshold. The theoretical curves are from Hempstead and Lax (Ref. 15). The experimental points $\Delta, \square$, and Oare from Gamo, Grace, and Walter (Ref. 23), Davidson and Mandel (Ref. 24), and Arecchi et al. (Ref. 35), respectively. Lower-case $p$ is the pump parameter of the model, $\langle\Lambda\rangle$ is the effective linewidth of intensity fluctuations,

$$
\langle\Lambda\rangle=\left\langle(\Delta \rho)^{2}\right\rangle / \int_{0}^{\infty}\langle\Delta \rho(t) \Delta \rho(0)\rangle d t,
$$

and $n^{(2)}$ is the normalized second factorial moment $\langle n(n-1)\rangle /\langle n\rangle^{2} \equiv\left\langle\omega^{2}\right\rangle$ of the photocount distribution $p(n, T)$ as $T \rightarrow 0$. In this limit, $n^{(2)}-1=\left\langle(\Delta \rho)^{2}\right\rangle /\langle\rho\rangle^{2}$ and $I / I_{0}$ is the light intensity normalized to threshold value $(p=0)$, i.e., $I / I_{0}=\langle\rho\rangle /\langle\rho\rangle_{\text {th }}=\langle\rho\rangle / 1.128$.

al contention that all well-designed oscillators behave like Van der Pol oscillators near threshold. ${ }^{15-18}$ The slowness of the intensity relaxation rate $\gamma / n^{\text {th }}$ is produced by a time scaling, ${ }^{16}$ and the smallness of cubic terms is produced by an amplitude scaling. ${ }^{15,18}$ The occurrence of this scaling for both ordinary oscillators (e.g., vacuum tube) and lasers near threshold is the analog of scaling that takes place in a phase transition near the critical temperature in which long-time long-wave fluctuations become of importance.

The simplest problem of laser statistics is the photocount problem for times so short (compared to the intensity correlation time) that the laser intensity does not change, so that the counts represent the steady-state photon or intensity distribution in the laser. Early measurements of this dis tribution by Freed and Haus ${ }^{19}$ and Smith and Armstrong $^{20}$ and of moments of this distribution by Arecchi, ${ }^{21}$ Chang et al.,${ }^{22}$ Gamo, Grace, and Walter, ${ }^{23}$ and Davidson and $\mathrm{Mandel}^{24}$ agree with the steady-state distribution for the Van der Pol model [Eq. (18B30) of the Brandeis lectures ${ }^{25}$ or Eqs. (7.18) and (7.21) of this paper.] See Fig. 1 for a comparison of the second factorial moment $n^{(2)}$ $=\langle m(m-1)\rangle /\langle m\rangle^{2}$ of the counting distribution with theory. A bibliography of initial experimental papers is given in Table III extending a similar table of Pike, ${ }^{26}$ and a related set of initiating theoretical papers is given in Table II in Sec. XI.

The intensity fluctuation spectrum was harder to compute since it involved two time averages. To overcome these difficulties, we introduced a quantum-regression theorem ${ }^{9}$ that related two-time averages to one-time averages and a dynamical correspondence $\mathrm{e}^{\mathbf{1 4 , 2 7 - 3 3}}$ between quantum random processes and associated classical random processes which permitted the translation of the problem into a classical stochastic problem. The intensity fluctuation spectrum, calculated for the associated classical Van der Pol oscillator by Hempstead and Lax ${ }^{15}$ and by Risken and Vollmer ${ }^{34}$ was found to be approximately Lorentzian with a linewidth possessing a minimum in the vicinity of threshold. Agreement displayed in Fig. 1 between the theoretical linewidth and experimental results of Gamo, Grace, and Walter, ${ }^{23}$ Arecchi et al. , ,5 and Davidson and $\mathrm{Mandel}^{24}$ gave further support to the Van der Pol model.

The laser spectrum (of amplitude and phase fluctuations) is also found to be Lorentzian with a linewidth that varies inversely with the laser power, and contains in addition a factor that varies from 2 to 1 as one goes from below to above threshold (see Fig. 2). By heterodyning two lasers against one another, measurements of the phase linewidth have been made by Hinckley and Freed ${ }^{36}$ and by Manes and Siegman. ${ }^{36}$ These measurements yield

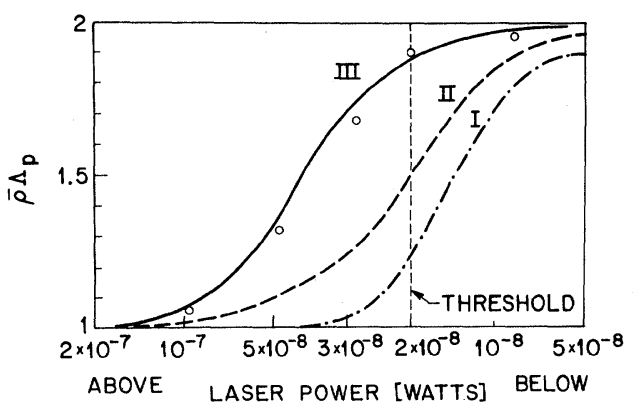

FIG. 2. Spectrum for phase and amplitude fluctuations of a laser is Lorentzian to a good approximation. The dimensionless (Ref. 15) half-width $\Lambda_{p}$ times the dimensionless (Ref. 15) mean power $\bar{\rho}$ are plotted against laser power. The experimental results are those of Gerhardt, Welling, and Güttner (Ref. 36). The theoretical curves I and II are due to Grosman and Richter (Ref. 34) and curve III to Risken (Ref. 34) and Hempstead and Lax (Ref. 15). 


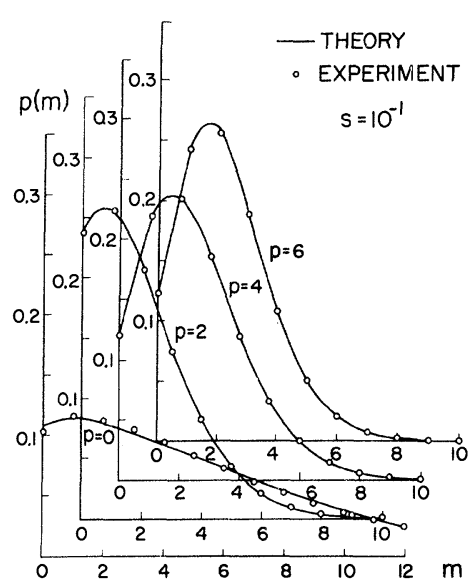

FIG. 3. Time-dependent photocount distribution $p(m, T)$ for a laser operated near threshold. The solid lines are the predictions of the present work (Ref. 38) and the circles correspond to measurements done by Meltzer, Davis, and Mandel (Ref. 39). Here $s=\langle\Lambda\rangle T$ $=T / T_{c}$ is the time in units of the correlation time (5.26).

a linewidth with the correct inverse power relationship, with a numerical coefficient in approximate quantitative agreement with theory. The results did not traverse the threshold region, however, and so did not display the predicted factorof -2 change in the coefficient. These results summarize earlier phase linewidth measurements. ${ }^{37}$

After this manuscript was submitted for publication, measurements using a Michelson interferometer technique (with multiple-reflection delay paths as long as $2 \mathrm{~km}$ ) were reported by Gerhardt, Welling, and Güttner. ${ }^{36}$ These measurements explore the threshold region displaying excellent agreement with the Risken ${ }^{34}$ and Hempstead and $\mathrm{Lax}^{15}$ calculations. These calculations which are exact solutions of the Fokker-Planck equation for a RWVP oscillator agree significantly better with the experimentally observed change in line-

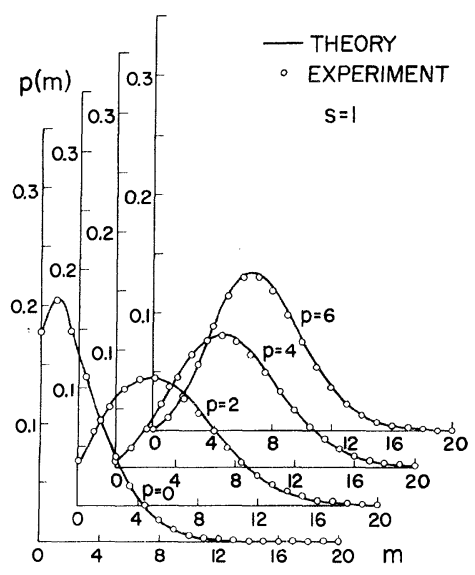

FIG. 4. Same as Fig. 3.

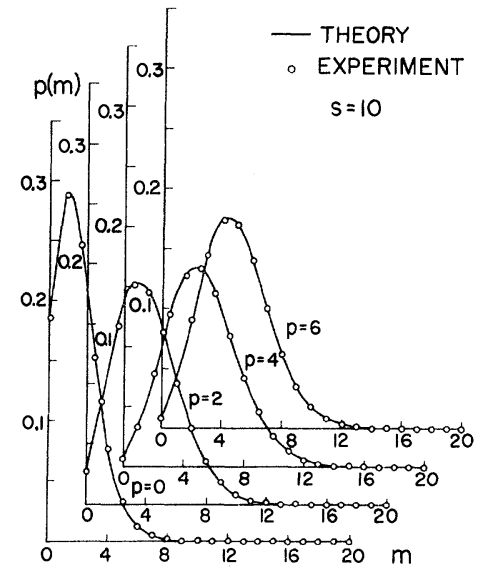

FIG. 5. Same as Fig. 3.

width in the threshold region than the attempt by Grossman and Richter ${ }^{34}$ at an approximate calculation in the spirit of the Landau theory of phase transitions.

The most detailed verification of the Van der Pol model, and the most difficult to achieve, is the photocount distribution for intermediate times, since this distribution involves multitime correlations of all orders. A preliminary report of our "exact" theoretical results ${ }^{38}$ stimulated measurements of $p(m, T)$ by Meltzer, Davis, and Mandel..$^{39}$ The excellent agreement between experiment (circles) and theory (solid wave) is displayed in Figs. 3-5. Measurements of the second-, third-, and fourth-normalized factorial moments of $p(m, T)$ were done by Jakeman et al..$^{40}$ and compared with theory in Figs. 6-8. The theoretical curves in Figs. 3-8 are calculated by the methods discussed in this paper.

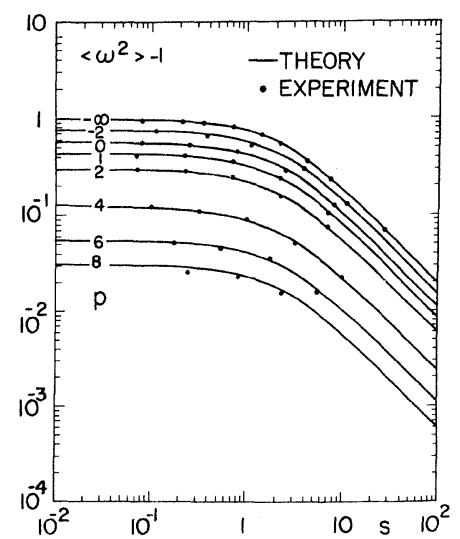

FIG. 6. Time-dependent normalized factorial moments $\left\langle\omega^{r}\right\rangle=n^{(r)} \equiv\left\langle m^{[r]}\right\rangle /\langle m\rangle^{r}$, where $\left\langle m^{[r]}\right\rangle=\langle m(m-1) \ldots$

$(m-r+1)\rangle$ are the $r$ th factorial moment. The solid lines are the predictions of the present work and the dats correspond to measurements done by Jakeman, Oliver, Pike, Lax, and Zwanziger (Ref. 40). 


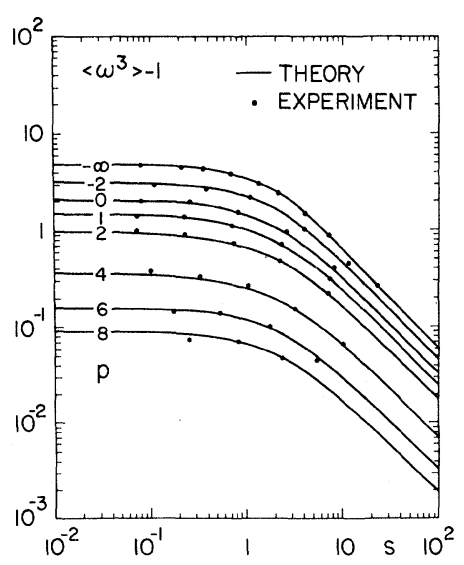

FIG. 7. Same as Fig. 6.

The successful calculations in this paper of the photocount distribution $p(m, T)$ are based on the Glauber photocount formula, ${ }^{41,42} \mathrm{Eq}$. (2.18). In our use of this formula, however, we find it es sential to describe the (positive frequency part of the) electric field $\overrightarrow{\mathrm{E}}^{+}$at the detector as proportional to the (quantum) amplitude $b(t)$ of the normal coordinate of the single mode in which the laser is operating. Thus, we use a single random amplitude that is not free but subject to the random fluctuations induced by spontaneous-emission effects, collisions, transmission losses, etc. The Glauber viewpoint, which has also been adopted by Mandel and Wolf, ${ }^{43}$ Klauder and Sudarshan, ${ }^{44}$ and Kelley and Kleiner, ${ }^{45}$ is that with the detector away from the source, the electric field behaves as a free field. This viewpoint is, of course, correct but it inhibits the usefulness of the formula. In the free-field case, the field must be expressed as an infinite linear combination of normal modes,

$$
\overrightarrow{\mathrm{E}}^{+}(r, t)=\sum_{k} \overrightarrow{\mathrm{u}}_{k}(r) a_{k}(t),
$$

each of which obeys the free-field time dependence

$$
a_{k}(t)=a_{k}(0) e^{-i \omega_{k} t} \text {. }
$$

This procedure achieves the desired aim of separating the source problem from the detector problem. Moreover, the time dependence of the intensity at the detector, $\overrightarrow{\mathrm{E}}^{-}\left(\overrightarrow{\mathrm{r}}_{0}, t\right) \cdot \overrightarrow{\mathrm{E}}^{+}\left(\overrightarrow{\mathrm{r}}_{0}, t\right)$, is entirely specified by Eqs. (1.1) and (1.2), and the problem is reduced to knowing the distribution of the initial values $a_{k}(0)$, i.e., the initial density operator $\rho(0)$ which is a function of the set of operators $\left\{a_{k}, a_{k}^{\dagger}\right\}$ or the corresponding Glauber-Sudarshan representation $P\left(\left\{\alpha_{k}, \alpha_{k}^{*}\right\}\right)$ in terms of the coherent states. Since $\omega_{k}=c k$, the amplitudes $a_{k}(0)$ are the individual Fourier components $b_{\omega}$ of $b(t)$ at $\omega$ $=c k$. Thus, the complete density operator $\rho\left(\left\{a_{k}, a_{k}^{\dagger}\right\}\right)$ describes all correlations of all Fourier components $b_{\omega}^{\dagger}$ and $b_{\omega}$, which is more information than required to solve our photodetection problem and more difficult to obtain.

Instead of dealing with the infinite number of free variables $\left\{a_{k}(t), a_{k}^{\dagger}(t)\right\}$, we deal with the single pair $b(t), b^{\dagger}(t)$ describing a Van der Pol oscillator in interaction with reservoirs that supply fluctuations as well as damping (and pumping). Since our solution of the photocount problem differs so markedly from that envisaged by Glauber, it is desirable to rederive, in the context of a nonlinear open system, the photocount formula Eq. (2.18) first given by Glauber ${ }^{41}$ and rederived by Kelley and Kleiner..$^{45}$ We shall not attempt to take account of attenuation effects in the detector, as discussed by Mollow, ${ }^{46}$ nor shall we idealize the photodetector as a harmonic oscillator to avoid perturbation techniques as has Glauber. ${ }^{42}$ However, in Sec. II we shall handle all combinatorial aspects of the problem reducing it to the question of calculating the quantum-mechanical probability of obtaining one count in each of $n$ infinitesimal intervals $\left(t_{j}, t_{j}+\Delta t_{j}\right)$ for $j=1,2, \ldots, n$ without the complicated requirement that no counts occur in between. The latter probability is calculated in the unexpurgated version ${ }^{47}$ of this paper by ordinary perturbational techniques without treating the electromagnetic field as a free field.

Section III describes the quantum classical correspondence that allows us to reduce our quantummechanical problem to an exactly equivalent classical one, and Sec. IV reviews the theory of the laser model. In Secs. V-VII, we deduce our methods for calculating photocount distributions and in Secs. VIII-X, we describe the numerical aspects of our work. A summary is given in Sec. XI.

\section{REVIEW OF PHOTODETECTOR THEORY}

Light detectors with some probability absorb a photon and emit an electron, which in a photo-

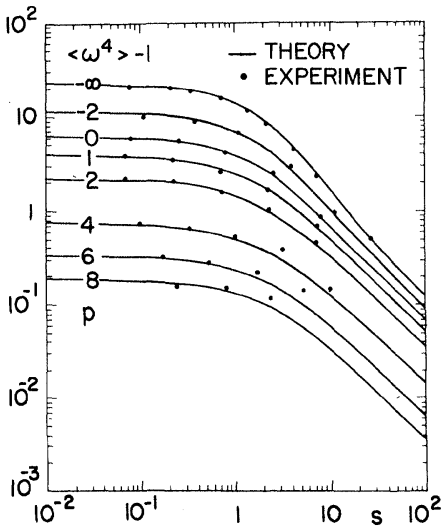

FIG. 8. Same as Fig. 6. 
multiplier results in a current pulse. A typical experiment consists of counting the number $m$ of pulses in a given time interval $T$. The photocount distribution function $p(m, T)$ describes a compounding of fluctuations in the light source with randomness in the detection processes. The aim of this section is to derive a formula for $p(m, T)$ which takes account of the Poisson character of detection fluctuations so that attention can be focused on the laser fluctuations.

An ideal photodetector is assumed to produce a sharp signal pulse (in time) for each photoabsorption, as compared to the observation time $T$. In addition, after each pulse, the ideal photodetector is assumed to reset itself instantaneously to the state it was in prior to the occurrence of the pulse. (Dead-time corrections are a separate problem considered by experimenters in reducing their data. $)^{48}$

\section{A. Classical Photodetectors}

The probability of registering a pulse in a small time interval $(T, T+\Delta T)$ is proportional to the time interval $\Delta T$, the photon field intensity ${ }^{49} \rho(T)$, and an efficiency factor $\epsilon$ that includes geometry as well as quantum efficiency. For an ideal classical photodetector, the probabilities $\epsilon \rho(T) \Delta T$ and $1-\epsilon \rho(T) \Delta T$ for registering or not registering a count are independent of previous events. With these assumptions, it follows immediately that the distribution $\hat{p}(m, T)$ which describes the detector photocount fluctuations for a fixed sample $\rho(t)$ of the possible source intensity fluctuation obeys a Poisson distribution, ${ }^{47}$

$$
\hat{p}(m, T)=(1 / m !) \Omega^{m} e^{-\Omega},
$$

where $\Omega$ is the time-integrated intensity variable

$$
\Omega=\epsilon \int_{0}^{T} \rho(t) d t
$$

The photocount distribution averaged over the random process $\rho(t)$ reduces to the for $m$ given by Mandel, ${ }^{50}$

$p(m, T) \equiv\langle\hat{p}(m, T)\rangle=\int\left(\Omega^{m} / m !\right) e^{-\Omega} W(\Omega) d \Omega$,

where the distribution of time-integrated intensities is

$$
W(\Omega)=\left\langle\delta\left(\Omega-\epsilon \int_{0}^{T} \rho(t) d t\right)\right\rangle .
$$

The mean number of counts equals the mean number of photons absorbed, and Eq. (2.2) gives

$$
\langle m\rangle=\langle\Omega\rangle=\epsilon\langle\rho\rangle T \text {. }
$$

Let us define a scaled variable $\omega$, the normalized time-integrated light intensity hitting the detector:

$$
\omega=\Omega /\langle\Omega\rangle=\int_{0}^{T} \rho(t) d t /\langle\rho\rangle T
$$

with distribution

$$
V(\omega)=\langle\Omega\rangle W(\Omega) .
$$

Equation (2.3) can be written

$$
p(m, T)=\int\left[(\langle m\rangle \omega)^{m} / m !\right] e^{-\langle m\rangle \omega} V(\omega) d \omega \text {. (2.8) }
$$

The normalized factorial moments $n^{(r)}(T)$ of $p(m$, $T)$, defined by $n^{(r)}(T) \equiv\left\langle m^{[r]}\right\rangle /\langle m\rangle^{r}$ or

$$
n^{(r)}(T)=\sum_{m} \frac{m(m-1) \ldots(m-r+1) p(m, T)}{\langle m\rangle^{r}},
$$

when evaluated by inserting Eq. (2.8) are found to be just the moments of $V(\omega)$ :

$$
n^{(r)}(T)=\int \omega^{r} V(\omega) d \omega=\left\langle\omega^{r}\right\rangle .
$$

This result explains why factorial moments are measured in order to characterize the statistical properties of the light source embodied in $V(\omega)$.

\section{B. Quantum-Photodetector Theory}

Kelley and Kleine ${ }^{45}$ break up the time interval $[0, T]$ into a set of $N$ intervals and calculate the probability $p(m, T)$ that counts occur in $m$ of these intervals, with no counts in the remaining intervals. Such "exclusive" probabilities are difficult to deal with. However, Kelley and Kleiner have shown that the exclusive nature of the probability is a combinatorial matter which can be handled separately. A simplified version of the Kelley-Kleiner proof can be given ${ }^{47}$ by direct use of the generating function

$$
M(\lambda, T)=\sum_{m}(1-\lambda)^{m} p(m, T)
$$

to show that

$$
\begin{array}{r}
M(\lambda, T)=\sum_{n=0}^{\infty} \frac{(-\lambda)^{n}}{n !} \int_{0}^{T} d t_{n} \ldots \int_{0}^{T} d t_{2} \int_{0}^{T} d t_{1} \\
\times w_{n}\left(t_{1}, t_{2}, \ldots, t_{n}\right),
\end{array}
$$

where $w_{n}\left(t_{1}, t_{2}, \ldots, t_{n}\right) \Delta t_{1} \Delta t_{2} \ldots \Delta t_{n}$ is the nonexclusive probability of a count in each of the $n$ intervals $\Delta t_{j}, j=1,2, \ldots, n$ with no restrictions on intervening events.

A variety of proofs have been given ${ }^{42,45}$ that

$$
w_{n}\left(t_{1}, t_{2}, \ldots, t_{n}\right)=\epsilon^{n}\left\langle b_{1}^{\dagger} b_{2}^{\dagger} \ldots b_{n}^{\dagger} b_{n} b_{n-1} \ldots b_{1}\right\rangle,
$$

where

$$
b_{j}^{\dagger} \equiv b^{\dagger}\left(t_{j}\right), \quad b_{j} \equiv b\left(t_{j}\right)
$$

are creation and destruction operators (at time $t_{j}$ ) for the laser mode and the \langle\rangle implies a trace against the density operator $\sigma_{F+S}(0)$ of the field with its sources and reservoirs (but not the detector). These proofs involve the use of perturbation theory (from $t_{j}$ to $t_{j}+\Delta t_{j}$ ) followed by the use of projection operators ${ }^{45,47}$ (from $t_{j}+\Delta t_{j}$ to $t_{j}+\Delta t_{j}^{+}$) 
on the detection apparatus. One finds

$$
\sigma_{F+s}\left(t_{j}+\Delta t_{j}^{*}\right)=\epsilon \Delta t_{j} b\left(t_{j}\right) \sigma_{F+s}\left(t_{j}\right) b^{\dagger}\left(t_{j}\right),
$$

where $\epsilon$ is an efficiency factor that involves the strength of coupling to the detector. The assumption is then made that if the system is not observed from $t_{j}+\Delta t_{j}^{+}$to $t_{j+1}$,

$$
\sigma_{F+s}\left(t_{j+1}\right)=\sigma_{F+s}\left(t_{j}+\Delta t_{j}^{+}\right) .
$$

This assumption neglects the reaction of the detector on the laser which is negligible in practice. Moreover, the photon loss due to the detector can be understood to be included in the loss parameter describing the laser (e.g., the inverse " $Q$ " of the laser mode).

It is further assumed that after each interaction the detector is restored to its initial state. The iteration of Eqs. (2.14) and (2.15) then leads to

$$
\begin{aligned}
\sigma_{F+S}\left(t_{n}+\Delta t_{n}^{+}\right) & =\epsilon^{n} \Delta t_{1} \Delta t_{2} \ldots \Delta t_{n} \\
& \times b_{n} \ldots b_{2} b_{1} \sigma_{F+S}(0) b_{1}^{\dagger} b_{2}^{\dagger} \ldots b_{n}^{\dagger},
\end{aligned}
$$

a result which under the trace operation reduces to $\mathrm{Eq}$. (2.13). If we let the symbol $T_{N}$ indicate that the operators following it are to be placed in normal order and in the apex time sequence shown in Eq. (2.13), Eqs. (2.12) and (2.13) can be combined into the simple form

$$
M(\lambda, T)=\left\langle T_{N} e^{-\lambda \Omega_{\text {OD }}}\right\rangle,
$$

so that

$$
\begin{aligned}
p(m, T) & =\left.\frac{1}{m !}\left(-\frac{\partial}{\partial \lambda}\right)^{m} M(\lambda, T)\right|_{\lambda=1} \\
& =\left\langle T_{N} \frac{\Omega_{\mathrm{op}}^{m}}{m !} e^{-\Omega_{\mathrm{op}}}\right\rangle,
\end{aligned}
$$

where

$$
\Omega_{\mathrm{OD}} \equiv \epsilon \int_{0}^{T} b^{\dagger}(t) b(t) d t .
$$

This is analogous to the corresponding classical results, Eq. (2.2). We shall refer to Eq. (2.18) as the Glauber photocount formula, ${ }^{41}$ since it was first stated and derived by him for the free-electromagnetic-field case. It is to be emphasized, however, that in the derivation discussed here ${ }^{47}$ we have not assumed that $b(t)$ is associated with a free field, or even a superposition of free-field modes. In the free-field case we would have $\left[b\left(t_{i}\right), b\left(t_{j}\right)\right]=0$, whereas in the derivation of our quantum rotating-wave oscillator, ${ }^{14}$ commutators between $b(t)$ and $b(t+\Delta t)-b(t)$ were found to be nonvanishing. Thus, in our case, the time-ordering operation in Eqs. (2.17) and (2.18) is essential, whereas it could have been ignored in the original Glauber derivation. $\}$

A formula that agrees with Eq. (2.18) for short times, but disagrees for larger times, has been derived by Scully and Lamb. ${ }^{51}$ The disagreement is not calculational but caused by their assumption of a model that "does not correspond to the familiar experimental situation." Their model uses the steady state of the laser as an initial condition, removes the lasing atoms from the cavity, and replaces them by detecting atoms. At long times, their detector depletes the photon supply. This is unrealistic experimentally, and leads at infinite times to unit quantum efficiency: if $n$ photons were in the cavity initially, all $n$ will be counted. Thus, the mean number of photocounts in a time $T$ saturates in this model, whereas it is linear in $T$ in the typical photodetector situation. Moreover, their results depend only on the initial laser density matrix and not at all on laser dynamics represented, for example, by the laser-intensity-fluctuation cor relation time.

\section{QUANTUM-CLASSICAL CORRESPONDENCE}

An exact correspondence between quantummechanical operators and $c$ numbers can be set up providing a definite ordering rule is adopted. The best-known correspondence, ${ }^{52}$ that of normal ordering (all $b^{\dagger}$ 's to the left of all $b^{\prime}$ 's), relates a $c$-number function $\bar{M}^{(n)}\left(\beta, \beta^{*}\right)$ to an operator $M^{(n)}\left(b, b^{\dagger}\right)$ in normal order by the normal ordering symbol $\mathfrak{n}$ which makes the replacements $\beta \rightarrow b, \beta^{*}$ $\rightarrow b^{\dagger}$ and places the result in normal order:

$$
\begin{aligned}
M\left(b, b^{\dagger}\right) & =M^{(n)}\left(b, b^{\dagger}\right) \equiv \mathfrak{N} \bar{M}^{(n)}\left(\beta, \beta^{*}\right) \\
& \equiv \int d^{2} \beta \bar{M}^{(n)}\left(\beta, \beta^{*}\right) \delta\left(\beta^{*}-b^{\dagger}\right) \delta(\beta-b),
\end{aligned}
$$

where $d^{2} \beta=d \operatorname{Re} \beta d \operatorname{Im} \beta$ and

$$
\delta\left(\beta^{*}-b^{\dagger}\right) \delta(\beta-b) \equiv\left(1 / \pi^{2}\right) \int e^{-\xi\left(\beta^{*}-\beta^{\dagger}\right)} e^{\xi^{*}(\beta-b)} d^{2} \xi .
$$

For example,

$$
b b^{\dagger}=b^{\dagger} b+1=\Re\left(|\beta|^{2}+1\right) .
$$

The connection between Eq. (3.1) and the GlauberSudarshan diagonal representation ${ }^{53,54}$ has been established, but the form (3.1) has the advantage that it can readily be generalized to many times. ${ }^{28}$ For example, if $\tau_{N}$ denotes replacing $c$ numbers by operators and placing the latter in normal order and in the apex time sequence of (2.13), we can set up the correspondence

$$
\begin{aligned}
M\left(b_{1}^{\dagger}, b_{2}^{\dagger}, \ldots, b_{n}^{\dagger} ; b_{n}, \ldots, b_{1}\right) & \\
= & \tau_{N} \bar{M}\left(\beta_{1}^{*}, \beta_{2}^{*}, \ldots, \beta_{n}^{*} ; \beta_{n}, \ldots, \beta_{1}\right) \\
= & \int d^{2} \beta_{1}, \ldots, d^{2} \beta_{n} \bar{M}\left(\beta_{1}^{*}, \ldots, \beta_{n}^{*} ; \beta_{n}, \ldots, \beta_{1}\right) \\
& \times \delta\left(\beta_{1}^{*}-b_{1}^{\dagger}\right), \ldots, \delta\left(\beta_{n}^{*}-b_{n}^{\dagger}\right) \delta\left(\beta_{n}-b_{n}\right), \ldots, \\
& \times \delta\left(\beta_{1}-b_{1}\right),
\end{aligned}
$$

where $b_{j} \equiv b\left(t_{j}\right)$ as in Eq. (2.13). The trace of Eq. (3.4) against the density matrix can be written

$\left\langle M\left(b_{1}^{\dagger}, \ldots, b_{n}^{\dagger} ; b_{n}, \ldots, b_{1}\right)\right\rangle$ 


$$
\begin{aligned}
=\int d^{2} \beta_{1}, \ldots, & d^{2} \beta_{n} \bar{M}\left(\beta_{1}^{*}, \ldots, \beta_{n}^{*} ; \beta_{n}, \ldots, \beta_{1}\right) \\
& \times P\left(\beta_{n}, \beta_{n}^{*}, t_{n} ; \ldots ; \beta_{1} \beta_{1}^{*}, t_{1}\right),
\end{aligned}
$$

where

$$
\begin{aligned}
P\left(\beta_{n}, \beta_{n}^{*}, t_{n} ; \ldots, \beta_{1} \beta_{1}^{*}, t_{1}\right) \equiv\left\langle\delta\left(\beta_{1}^{*}-b_{1}^{\dagger}\right), \ldots,\right. \\
\left.\quad \times \delta\left(\beta_{n}^{*}-b_{n}^{\dagger}\right) \delta\left(\beta_{n}-b_{n}\right), \ldots, \delta\left(\beta_{1}-b_{1}\right)\right\rangle .
\end{aligned}
$$

Equation (3.5) tells us that provided our operators are in the chosen normal-ordered apex time sequence, quantum-mechanical averages can be replaced by associated $c$-number averages which we shall denote \langle\rangle$_{c}$. Since

$$
\begin{aligned}
& T_{N} e^{-\lambda \Omega_{\mathrm{oD}}}=\tau_{N} e^{-\lambda \Omega}, \\
& T_{N}\left(\Omega_{\mathrm{OD}}^{m} / m !\right) e^{-\lambda \Omega_{\mathrm{op}}}=\tau_{N}\left(\Omega^{m} / m !\right) e^{-\lambda \Omega},
\end{aligned}
$$

where

$$
\Omega \equiv \epsilon \int_{0}^{T}|\beta(t)|^{2} d t,
$$

the characteristic function and desired counting distribution can be obtained from

$$
\begin{aligned}
& M(\lambda, T)=\left\langle e^{-\lambda \Omega}\right\rangle_{c}, \\
& p(m, T)=\left\langle\left(\Omega^{m} / m !\right) e^{-\Omega}\right\rangle_{c},
\end{aligned}
$$

even though averages over an arbitrarily large number of times appear when the exponential is expanded.

Any function $F(\Omega)$ of the time-integrated intensity $\Omega$ such as in Eq. (3.10) or (3.11) can be rewritten as an integral

$$
\langle F(\Omega)\rangle_{c}=\int F(\Omega) W(\Omega) d \Omega,
$$

where the work has been transferred to finding the distribution of intensities,

$$
W(\Omega)=\left\langle\delta\left(\Omega-\epsilon \int_{0}^{T}|\beta(t)|^{2} d t\right)\right\rangle_{c} .
$$

Equations (3.10) and (3.11) apply whether or not the photon system alone can be regarded as Markoffian. We have shown, however, that if the electromagnetic system is Markoffian in the sense that the regression theorem is obeyed, ${ }^{28}$ the $n$-time pseudoprobability factorizes,

$$
\begin{aligned}
P\left(\beta_{n}, \beta_{n}^{*}, t_{n}, \ldots, \beta_{1}, \beta_{1}^{*}, t_{1}\right)^{\prime} \\
=P\left(\beta_{n}, \beta_{n}^{*}, t_{n} \mid \beta_{n-1}, \beta_{n-1}^{*}, t_{n-1}\right) \\
\quad \times \cdots P\left(\beta_{3}, \beta_{3}^{*}, t_{3} \mid \beta_{2}, \beta_{2}^{*}, t_{2}\right) \\
\quad \times P\left(\beta_{2}, \beta_{2}^{*}, t_{2} \mid \beta_{1}, \beta_{1}^{*}, t_{1}\right) P\left(\beta_{1}, \beta_{1}^{*}, t_{1}\right),
\end{aligned}
$$

showing that multitime averages can be calculated with use of products of two-time conditional probabilities just as for an ordinary classical Markoffian process. Moreover, $P\left(\beta, \beta^{*}, t \mid \beta_{0}, \beta_{0}^{*}, t_{0}\right)$ is that solution of the equation of motion obeyed by the one-time distribution

$$
P\left(\beta, \beta^{*}, t\right)=\left\langle\delta\left[\beta^{*}-b^{\dagger}(t)\right] \delta[\beta-b(t)]\right\rangle,
$$

which reduces to $\delta\left(\beta^{*}-\beta_{0}^{*}\right) \delta\left(\beta-\beta_{0}\right)$ at $t=t_{0}$. (i. e., the Green's function).

\section{LASER THEORY}

A laser model in which a single electromagnetic mode couples equivalently (except for phase) to many atoms is depicted in Fig. 9. Field losses and atomic collisions are produced by reservoirs. Instead of referring to all authors who have studied this model, we indicate in Ref. 55 reviews written by proponents of the three most active groups in this field: Haken of Stuttgart, Lamb and Scully of Yale and the University of Arizona, and Lax of Bell Telephone Laboratories and City College and their co-workers.

For the case of a homogeneously broadened line, after removal of certain phase factors, the field couples in a symmetrical way to all atoms. Thus a closed description can be given in terms of six variables: the field variables $b$ and $b^{\dagger}$, the total upper- and lower-state populations $N_{2}$ and $N_{1}$, and the transition polarization variables $M$ and $M^{\dagger}$. For this reason the ansatz for the density matrix of a laser made by Gordon ${ }^{32}$ and the six-variable description of Lax and Yuen ${ }^{33}$ are exact.

As discussed in Sec. I, only for the region near threshold are powerful techniques required. Fortunately, in that region the long time scale of the fluctuations (increased by a factor $n^{\text {th }} \sim 10^{4}$ over the usual decay times) permits the adiabatic elimination of all but two variables. The result is an equation of motion for the density operator $\rho(b$, $\left.b^{\dagger}, t\right)$ or the associated pseudoprobability $P\left(\beta, \beta^{*}\right.$, t) defined in Eq. (3.13).

Moreover, the long time scale means that all interactions with atomic variables take place so rapidly compared to the relevant times that these variables can be adiabatically eliminated leaving a Markoffian process for the field variables $b(t)$, $b^{\dagger}(t)$ or the associated "classical" random process

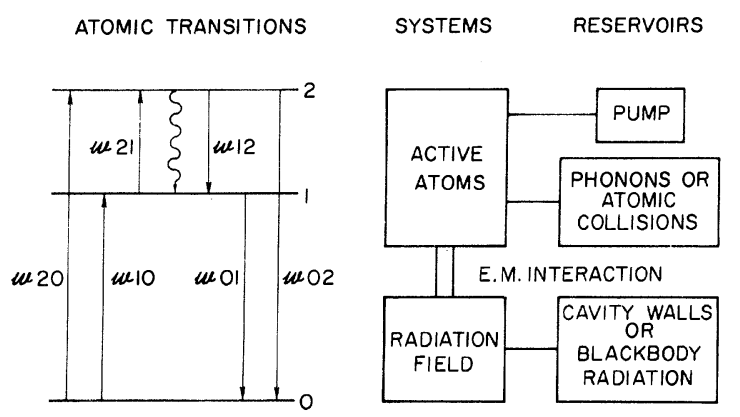

FIG. 9. Laser model. Radiative transitions (wavy arrow) are induced by the dynamic atom-field coupling. Nonradiative transitions (straight arrows) and quantumnoise sources are derivable consequences of the coupling to the reservolrs. 
$\beta(t), \beta(t)^{*}$ described by $P\left(\beta, \beta^{*}, t\right)$. This random process can equally well be described as a Langevin or noise-source process. In this form, we have established ${ }^{56}$ that near threshold a homogeneously broadened laser is describable as a RWVP oscillator:

$$
\frac{d \beta}{d t}=\left[\frac{1}{2} \gamma-i\left(\omega_{0}-\omega_{c}\right)\right]\left(\Pi-R_{0}|\beta|^{2}\right) \beta+F_{\beta}(t),
$$

where $\gamma$ is the cold-cavity decay rate, $\omega_{0}$ the resonant frequency, II an effective pump rate, and $R_{0}$ a saturation parameter. ${ }^{56}$ The noise sources $F_{\beta}$ and $F_{\beta}^{*}$ are treated as approximately Gaussian with

$$
\left\langle F_{\beta}(t) * F_{\beta}(u)\right\rangle=2 D_{\beta} *_{\beta} \delta(t-u)
$$

(and the complex-conjugate equation) as the only nonvanishing second moments. The parameters $\Pi, R_{0}$, and $D_{\beta} *_{\beta}$ are constants which depend on the particular model assumed for a laser, e.g., homogeneous or inhomogeneous, and depend on pump rates, decay constants, etc. We have previously given reasons for expecting such an RWVP form for any well-designed single-frequency oscillator ${ }^{15,18}$ including vacuum-tube oscillators. By a combination of amplitude and time scaling,

$$
\beta=\xi \beta^{\prime}, t=T t^{\prime},
$$

our RWVP model can be reduced for the in-tune case to the canonical scaled form

$$
\begin{aligned}
& \frac{d \beta^{\prime}}{d t^{\prime}}=\left(p-\left|\beta^{\prime}\right|^{2}\right) \beta^{\prime}+F_{\beta^{\prime}}\left(t^{\prime}\right), \\
& \left\langle F_{\beta^{\prime}}\left(t^{\prime}\right) * F_{\beta^{\prime}}\left(u^{\prime}\right)\right\rangle=4 \delta\left(t^{\prime}-u^{\prime}\right) .
\end{aligned}
$$

If we introduce intensity and phase variables $\rho$, $\varphi$,

$$
\beta^{\prime}=\rho^{1 / 2} e^{-i \varphi},
$$

and drop the prime from $t^{\prime}$, the equations for $\beta^{\prime}$ and $\left(\beta^{\prime}\right) *$ transform to ${ }^{57}$

$$
\begin{aligned}
& \frac{d \rho}{d t}=A(\rho)+F_{\rho}(t), \\
& A(\rho)=2 p \rho-2 \rho^{2}+4, \\
& \frac{d \varphi}{d t}=F_{\varphi}(t), \\
& \left\langle F_{\rho}(t) F_{\rho}(u)\right\rangle=2 D_{\rho \rho} \delta(t-u), \\
& D_{\rho \rho} \equiv D(\rho)=4 \rho, \\
& \left\langle F_{\varphi}(t) F_{\varphi}(u)\right\rangle=2 D_{\varphi \varphi} \delta(t-u), \\
& D_{\varphi \varphi}=(1 / \rho), \\
& \left\langle F_{\rho}(t) F_{\varphi}(u)\right\rangle=0 .
\end{aligned}
$$

Corresponding to the Langevin-noise-source equations (4.6) and (4.8), there is the associated Fokker-Planck form ${ }^{57,58}$ :

$$
\begin{aligned}
& \frac{\partial P(\rho, \varphi, t)}{\partial t}=-\frac{L P(\rho, \varphi, t)}{\partial t}, \\
& -L=-\frac{\partial}{\partial \rho} A(\rho)+\frac{\partial^{2}}{\partial \rho^{2}} D(\rho)+\frac{\partial^{2}}{\partial \varphi^{2}} D_{\varphi \varphi} \\
& \quad=-\frac{\partial}{\partial \rho}\left(2 p \rho-2 \rho^{2}+4\right)+\frac{\partial^{2}}{\partial \rho^{2}}(4 \rho)+\frac{1}{\rho} \frac{\partial^{2}}{\partial \varphi^{2}} .
\end{aligned}
$$

When we are concerned entirely with intensity fluctuations, as we shall be in this paper, the pseudoprobability $P(\rho, \varphi, t)$ can be assumed independent of $\varphi$ and the last term in $L$ omitted [see Eqs.

(5.18) and (5.19)].

\section{PROBABILITY DISTRIBUTION $V(\omega)$}

Since we cannot directly evaluate the desired distribution function

$$
W(\Omega) \equiv\left\langle\delta\left[\Omega-\epsilon \int_{0}^{T} \rho(t) d t\right]\right\rangle,
$$

we calculate instead its Laplace transform

$$
M\left(\lambda^{\prime}\right) \equiv \int_{0}^{T} e^{-\lambda^{\prime} \Omega} W(\Omega) d \Omega
$$

for real $\lambda^{\prime}$, and in Secs. VIII and IX invert this Laplace transform numerically to obtain $W(\Omega)$.

It is convenient to work with the scaled variable

$$
\omega=\Omega /\langle\Omega\rangle=\int_{0}^{T} \rho(t) d t /\langle\rho\rangle T,
$$

which has a distribution

$$
V(\omega)=\langle\Omega\rangle W(\Omega)
$$

that is independent of the efficiency parameter $\epsilon$ :

$$
V(\omega)=\left\langle\delta\left[\omega-(1 /\langle\rho\rangle T) \int_{0}^{T} \rho(t) d t\right]\right\rangle .
$$

By writing $\lambda^{\prime}=\lambda / \epsilon$, Eq. (5.4) transforms into

$$
\begin{aligned}
M(\lambda) & =\int e^{-\lambda\langle\rho\rangle T \omega} V(\omega) d \omega \\
& =\left\langle\exp \left(-\lambda \int_{0}^{T} \rho(t) d t\right)\right\rangle .
\end{aligned}
$$

Following techniques used by Kac and Siegert, ${ }^{59}$ $\mathrm{Lax}^{60}$ showed that for random Markoffian processes in a set of variables $\vec{a}, \rho(\vec{a})$ being an arbitrary functions of $\vec{a}$, the path-integral average, Eq. (5.6), can be calculated from

$$
\begin{aligned}
& M(\lambda)=\int d \overrightarrow{\mathrm{a}}_{0} P_{0}\left(\overrightarrow{\mathrm{a}}_{0}\right) \int d \overrightarrow{\mathrm{a}} \hat{P}\left(\overrightarrow{\mathrm{a}}, t \mid \overrightarrow{\mathrm{a}}_{0}, t_{0}\right), \\
& t-t_{0}=T \geq 0 \text {. }
\end{aligned}
$$

Here the Markoff random process $\vec{a}(t)$ has a probability distribution $P(\overrightarrow{\mathrm{a}}, t)$ which obeys

$$
\frac{\partial P(\overrightarrow{\mathrm{a}}, t)}{\partial t}=-L P(\overrightarrow{\mathrm{a}}, t)
$$

where $L$ is a linear differential or integral operator, and

$$
L P_{0}(\vec{a})=0
$$

defines the steady state of this random process. The probability $\hat{P}$ describes the same process modified by a loss rate $-\lambda \rho(\overrightarrow{\mathrm{a}}) \hat{P}$ : 


$$
\frac{\partial \hat{P}}{\partial t}=-(L+\lambda \rho) \hat{P},
$$

and Eq. (5.7) makes use of a conditional probability, or Green's-function solution of Eq. (5.10), that obeys the initial condition

$$
\hat{P}\left(\overrightarrow{\mathrm{a}}, t_{0} \mid \overrightarrow{\mathrm{a}}_{0}, t_{0}\right)=\delta\left(\overrightarrow{\mathrm{a}}-\overrightarrow{\mathrm{a}}_{0}\right) \text {. }
$$

$L$ is usually a non-Hermitian operator in the Sturm-Liouville sense. We will assume that $L$ and its Hermitian adjoint $L^{\dagger}$ have complete sets of biorthogonal eigenfunctions, ${ }^{61}$

$$
\begin{aligned}
& L P_{j}=\Lambda_{j} P_{j} ; \quad L^{\dagger} \varphi_{i}=\Lambda_{i}^{*} \varphi_{i}, \\
& \left(\varphi_{i}, P_{j}\right)=\int d \overrightarrow{\mathrm{a}} \varphi_{i}^{*}(\overrightarrow{\mathrm{a}}) P_{j}(\overrightarrow{\mathrm{a}})=\delta_{i j} .
\end{aligned}
$$

We also assume we can obtain the eigenfunctions of the modified operator $L+\lambda \rho$,

$$
\begin{aligned}
& (L+\lambda \rho) \hat{P}_{j}=\hat{\Lambda}_{j} \hat{P}_{j} ; \\
& \left(L^{\dagger}+\lambda^{*} \rho^{*}\right) \hat{\varphi}_{i}=\hat{\Lambda}_{i}^{*} \hat{\varphi}_{i}, \quad\left(\hat{\varphi}_{i}, \hat{P}_{j}\right)=\delta_{i j} .
\end{aligned}
$$

The Green's-function solution of Eqs. (5.10) and (5.11) can be written ${ }^{61,62}$

$$
\hat{P}\left(\overrightarrow{\mathrm{a}}, t \mid \overrightarrow{\mathrm{a}}_{0}, t_{0}\right)=\sum_{n} e^{-\hat{\Lambda}_{n} T} \hat{P}_{n}(\overrightarrow{\mathrm{a}}) \hat{\varphi}_{n}^{*}\left(\overrightarrow{\mathrm{a}}_{0}\right), \quad t-t_{0}=T \geq 0 .
$$

We show in Appendix A that for random processes obeying time reversal and stationarity,

$$
\begin{aligned}
& \varphi_{n}^{*}(\overrightarrow{\mathrm{a}})=P_{n}\left(\overrightarrow{\mathrm{a}}^{T}\right) / P_{0}(\overrightarrow{\mathrm{a}}), \\
& \hat{\varphi}_{n}^{*}(\overrightarrow{\mathrm{a}})=\hat{P}_{n}\left(\overrightarrow{\mathrm{a}}^{T}\right) / P_{0}(\overrightarrow{\mathrm{a}}),
\end{aligned}
$$

when $a_{j}^{T}= \pm a_{j}$ according to whether $a_{j}$ is even or odd under time reversal. Inserting Eqs. (5.16) and (5.17) in Eq. (5.7) gives us our basic working formula:

$$
M(\lambda)=\sum_{n} e^{-\hat{\Lambda}_{n} T}\left[\int \hat{P}_{n}(\overrightarrow{\mathrm{a}}) d \overrightarrow{\mathrm{a}}\right]^{2} .
$$

For any laser model, $M(\lambda)$ yields $V(\omega)$ through the inverse of Eq. (5.6), and $V(\omega)$ gives $p(m, T)$ through Eq. (2.8).

The operator $L$ for the laser model we are considering, given by Eq. (4.13), has eigenfunctions of the form $f(\rho) e^{i l \varphi}$. The integral over $\varphi$ in Eq. (5.18) assures that only the $l=0$ (amplitude-fluctuation) modes contribute to $p(m, T)$. We can thus restrict our attention to

$$
L=-\frac{\partial}{\partial \rho}\left(2 \rho^{2}-2 p \rho-4\right)-\frac{\partial^{2}}{\partial \rho^{2}}(4 \rho) .
$$

Alternatively, we could have arrived at this result directly by arguing that $M(\lambda)$ in Eq. (5.6) involves the statistics of $\rho$ but not $\varphi$.

The eigenfunctions of $L$ and $L+\lambda \rho$ of Eq. (5.19) cannot be found in simple closed form, and a numerical approach is necessary. One can solve for the eigenfunctions $\hat{P}_{n}(\rho)$ of $L+\lambda \rho$ in terms of our previously computed eigenfunctions ${ }^{15} P_{n}(\rho)$ of $L$ by setting

$$
\hat{P}_{n}(\rho)=\sum_{j} P_{j}(\rho) C_{j n}
$$

in Eq. (5.14) to obtain secular equations for $C_{j n}$ and $\hat{\Lambda}_{n}$ :

$$
\sum_{j}\left[\left(\Lambda_{i}-\hat{\Lambda}_{n}\right) \delta_{i j}+\lambda \rho_{i j}\right] C_{f n}=0 \quad \text { for all } i,
$$

where

$$
\rho_{i j}=\int d \rho P_{i}(\rho) \rho P_{j}(\rho) / P_{0}(\rho) .
$$

From Eq. (5.17), $\varphi_{0}^{*}=1$, and Eq. (5.13) gives

$$
\int d \rho P_{j}(\rho)=\delta_{0 j} \text {. }
$$

The insertion of $(5.20)$ and $(5.23)$ into $(5.18)$ yields for $M(\lambda)$,

$$
M(\lambda)=\sum_{n} \exp \left(-s \hat{\Lambda}_{n} /\langle\Lambda\rangle\right)\left(C_{0 n}\right)^{2},
$$

a result that requires no knowledge of $P_{n}(\rho)$ other than the matrix elements $\rho_{i j}$ of Eq. (5.22). The matrix elements $\rho_{i j}$ as a function of operating point $p$ are available from one of us (M. L.) on request, but are omitted to save space.

Here we have introduced the dimensionless time

$$
s=\langle\Lambda\rangle T,
$$

where $\langle\Lambda\rangle$ is the linewidth of the spectrum of intensity fluctuations,

$$
\begin{aligned}
& \langle\Lambda\rangle^{-1}=T_{c}=\left\langle(\Delta \rho)^{2}\right\rangle^{-1} \int_{0}^{\infty}\langle\Delta \rho(t) \Delta \rho(0)\rangle d t, \\
& \Delta \rho=\rho(t)-\langle\rho\rangle,
\end{aligned}
$$

known $n^{15,34}$ to be approximately Lorentzian, and thus given by the ratio of the area to $\pi$ times the height of the spectrum. $\langle\Lambda\rangle$ is referred to by other authors as $\Lambda_{\text {eff }}$ for effective $\Lambda$; it can be expressed as a superposition of the eigenvalues $\Lambda_{i}$,

$$
\langle\Lambda\rangle^{-1} \equiv \Lambda_{\text {eff }}^{-1}=\sum_{n} \frac{p_{n}}{\Lambda_{n}},
$$

where the weights

$$
p_{n}=p_{0, n}=\left(\rho_{0 n}\right)^{2} / \sum_{m \neq 0} \rho_{0 m}^{2}
$$

and the eigenvalues $\Lambda_{n}=\Lambda_{0, n}$ have been computed by Hempstead and $\operatorname{Lax}^{15}$ and Risken and Vollmer. ${ }^{34}$

Equation $(5.6)$ can be written as a Laplace transform,

$$
\begin{aligned}
M(\lambda)=\bar{M}(\nu) & =\int_{0}^{\infty} e^{-\nu \omega} V(\omega) d \omega \\
& =\left\langle\exp \left[-(\nu / s) \int_{0}^{s} \sigma(t) d t\right]\right\rangle,
\end{aligned}
$$

where

$\sigma(t) \equiv \rho(t) /\langle\rho\rangle, \quad \nu=\lambda\langle\rho\rangle T=\lambda\langle\rho\rangle s /\langle\Lambda\rangle$.

Equation (5.21), with the change of variables of Eq. (5.30), becomes

$$
\sum_{j}\left[\left(\Lambda_{i}-\hat{\Lambda}_{n}\right) \delta_{i j}+(\nu\langle\Lambda\rangle /\langle\rho\rangle s) \rho_{i j}\right] C_{f n}=0,
$$


TABLE I. Eigenvalues of the Fokker-Planck operator $L=-(\partial / \partial \rho)\left(2 \rho^{2}-2 p \rho-4\right)-\left(\partial^{2} / \partial \rho^{2}\right)(4 \rho)$ corresponding to the RWVP laser model, Eq. (5.19), in the text for various values of $p$. The lowest eigenvalue $\Lambda_{0}=0$ in each case is not listed. The remaining $\Lambda_{n}$ increase monotonically with $n$. The results for $n=1,2, \ldots, 10$ are given sequentially. 〈lambda〉 stands for the harmonic-mean $\langle\Lambda\rangle$ defined in Eq. (5.26).

\begin{tabular}{ccccc}
\hline$p=-10$ & $p=-2$ & $p=-1$ & $p=0$ & $p=1$ \\
\hline $2.1469 \times 10$ & 7.878 & 6.6358 & 5.6266 & 4.9284 \\
$4.4871 \times 10$ & $1.8948 \times 10$ & $1.6498 \times 10$ & $1.4363 \times 10$ & $1.2604 \times 10$ \\
$6.9954 \times 10$ & $3.2312 \times 10$ & $2.8688 \times 10$ & $2.5452 \times 10$ & $2.2664 \times 10$ \\
$9.6546 \times 10$ & $4.7573 \times 10$ & $4.2791 \times 10$ & $3.8461 \times 10$ & $3.4642 \times 10$ \\
$1.2452 \times 10^{2}$ & $6.4487 \times 10$ & $5.8559 \times 10$ & $5.3139 \times 10$ & $4.8287 \times 10$ \\
$1.5377 \times 10^{2}$ & $8.2888 \times 10$ & $7.5821 \times 10$ & $6.9314 \times 10$ & $6.3427 \times 10$ \\
$1.8421 \times 10^{2}$ & $1.0265 \times 10^{2}$ & $9.4451 \times 10$ & $8.6859 \times 10$ & $7.9935 \times 10$ \\
$2.1579 \times 10^{2}$ & $1.2368 \times 10^{2}$ & $1.1435 \times 10^{2}$ & $1.0567 \times 10^{2}$ & $9.7711 \times 10$ \\
$2.4843 \times 10^{2}$ & $1.4589 \times 10^{2}$ & $1.3544 \times 10^{2}$ & $1.2568 \times 10^{2}$ & $1.1668 \times 10^{2}$ \\
$2.8210 \times 10^{2}$ & $1.6922 \times 10^{2}$ & $1.5764 \times 10^{2}$ & $1.4681 \times 10^{2}$ & $1.3676 \times 10^{2}$ \\
$\langle$ lambda $\rangle$ & $\langle$ lambda $\rangle$ & $\langle$ lambda $\rangle$ & $\langle$ lambda $\rangle$ & $\langle$ lambda $\rangle$ \\
$2.1479 \times 10$ & 7.9889 & 6.7927 & 5.8539 & 5.2688 \\
$p=2$ & & & & \\
4.6358 & 5.6976 & 9.4499 & $1.4651 \times 10$ & $1.9114 \times 10$ \\
$1.1286 \times 10$ & $1.0236 \times 10$ & $1.1582 \times 10$ & $1.4967 \times 10$ & $1.9124 \times 10$ \\
$2.0387 \times 10$ & $1.7657 \times 10$ & $1.8059 \times 10$ & $2.3666 \times 10$ & $3.4518 \times 10$ \\
$3.1396 \times 10$ & $2.6900 \times 10$ & $2.5614 \times 10$ & $2.8389 \times 10$ & $3.5394 \times 10$ \\
$4.4064 \times 10$ & $3.7774 \times 10$ & $3.4882 \times 10$ & $3.6289 \times 10$ & $4.4496 \times 10$ \\
$5.8220 \times 10$ & $5.0111 \times 10$ & $4.5578 \times 10$ & $4.5398 \times 10$ & $5.0780 \times 10$ \\
$7.3738 \times 10$ & $6.3789 \times 10$ & $5.7588 \times 10$ & $5.5858 \times 10$ & $5.9745 \times 10$ \\
$9.0520 \times 10$ & $7.8714 \times 10$ & $7.0820 \times 10$ & $6.7524 \times 10$ & $6.9813 \times 10$ \\
$1.0849 \times 10^{2}$ & $9.4809 \times 10$ & $8.5200 \times 10$ & $8.0320 \times 10$ & $8.1069 \times 10$ \\
$1.2757 \times 10^{2}$ & $1.1201 \times 10^{2}$ & $1.0067 \times 10^{2}$ & $9.4184 \times 10$ & $9.3398 \times 10$ \\
$\langle$ lambda $\rangle$ & $\langle$ lambda $\rangle$ & $\langle$ lambda $\rangle$ & $\langle$ lambda $\rangle$ & $\langle$ lambda $\rangle$ \\
5.1750 & 7.1122 & $1.1231 \times 10$ & $1.5462 \times 10$ & $1.9582 \times 10$ \\
\hline \hline
\end{tabular}

or else

$$
\sum_{j}\left[\left(E_{i}-\hat{E}_{n}\right) \delta_{i f}+\mu \rho_{i f}\right] C_{j n}=0
$$

with

$$
\begin{aligned}
& \mu=\nu /\langle\rho\rangle s=\lambda /\langle\Lambda\rangle, \\
& E_{i} \equiv \Lambda_{i} /\langle\Lambda\rangle, \quad \hat{E}_{n} \equiv \hat{\Lambda}_{n} /\langle\Lambda\rangle .
\end{aligned}
$$

Equation (5.24) now reads

$$
M(\lambda)=\bar{M}(\nu)=\sum_{n} e^{-\hat{E}_{n}(\mu) s}\left[C_{0 n}(\mu)\right]^{2} .
$$

Equations (5.29), (5.32), and (5.34) are especially useful since sets of $\Lambda_{i}$ and $\rho_{i j}$ have already been computed by Hempstead and Lax ${ }^{15,63}$ for a range of values of the pump parameter $p$. Values of $\Lambda_{i}$ and $\langle\Lambda\rangle$ are listed ${ }^{47}$ in Table I. The previously unpublished values of $\rho_{i j}$ (see Ref. 47) were used in the present work to compute $\bar{M}(\nu)$. Alternatively, one can numerically integrate the perturbed eigenvalue equation $(5.14)$ and work with Eq. (5.18).

The inverse Laplace transform of $\bar{M}(\nu), V(\omega)$, was computed as described in Sec. IX. The probability distributions $V(\omega)$ obtained are shown in Figs. 10-12 for a range of values of $p$ and $s$.

The procedure by which we have reduced the problem to a purely radial problem is not re-

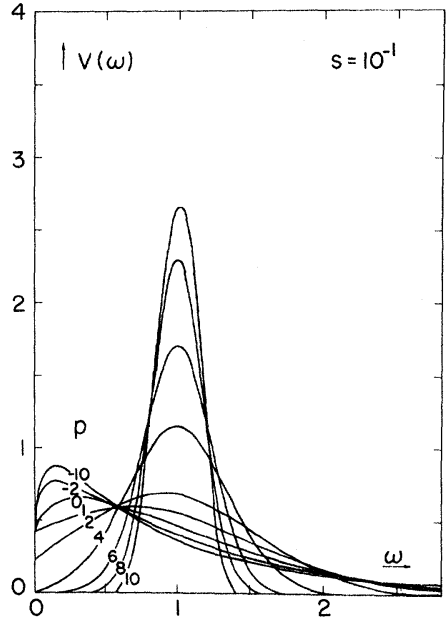

FIG. 10. Probability distributions $V(\omega)$ of the normalized $(\langle\omega\rangle=1)$ integrated light intensity $\omega$ computed from the RWVP oscillator model of a laser operating near threshold, for various values of the pump $p$ and the scaled time $s$. See Table V (Ref. 47) for more complete results.

stricted to the van der Pol oscillator. More general nonlinearities are possible. The factorization of eigenfunctions into the form $P_{l n}(\rho) e^{i l \varphi}$ does require, however, that all the drift vectors and diffusion coefficients be independent of $\varphi$, although they can remain arbitrary functions of $\rho$.

\section{MOMENTS OF DISTRIBUTION $V(\omega)$}

For comparison with the factorial-moment $n^{(r)}$ data of Jakeman et al. , ${ }^{40}$ we shall in view of Eq. (2.10) obtain the exact moments $\left\langle\omega^{r}\right\rangle$ of $V(\omega)$. From Eq. (5.29), one derives

$$
(-1)^{r}\left(\frac{\partial^{r}}{\partial \nu^{r}} \bar{M}(\nu)\right)_{\nu=0}=\int \omega^{r} V(\omega) d \omega=\left\langle\omega^{r}\right\rangle .
$$

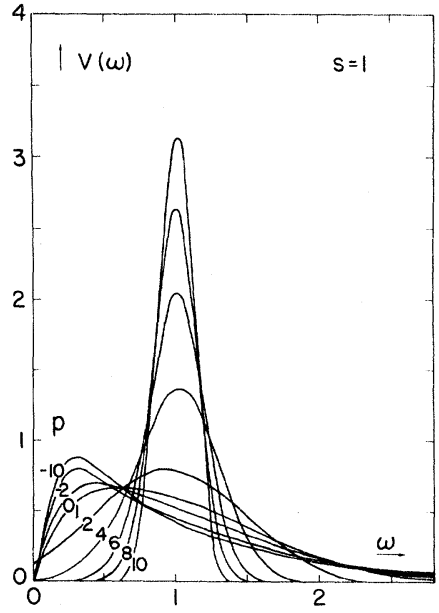

FIG. 11. Same as Fig. 10. 


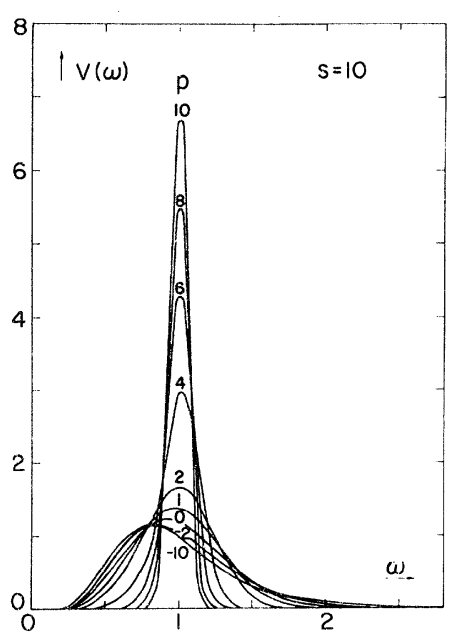

FIG. 12. Same as Fig. 10.

Equation (5.30) gives the identity

$$
\frac{\partial^{r}}{\partial \nu^{r}} \bar{M}(\nu)=\left(\frac{1}{\langle\rho\rangle T}\right)^{r} \frac{\partial^{r}}{\partial \lambda^{r}} M(\lambda) \text {. }
$$

We expand the derivatives of $\hat{P}_{n}(\rho)$ in terms of $\hat{P}_{n}(\rho)$ :

$$
\begin{aligned}
& \frac{\partial}{\partial \lambda} \hat{P}_{n} \equiv \hat{P}_{n}^{\prime}=\sum_{m} \hat{P}_{m} D_{m n}, \\
& \frac{\partial^{2}}{\partial \lambda^{2}} \hat{P}_{n} \equiv \hat{P}_{n}^{\prime \prime}=\sum_{m} \hat{P}_{m} E_{m n}, \\
& \frac{\partial^{3}}{\partial \lambda^{3}} \hat{P}_{n} \equiv \hat{P}_{n}^{\prime \prime \prime}=\sum_{m} \hat{P}_{m} F_{m n}, \\
& \frac{\partial^{4}}{\partial \lambda^{4}} \hat{P}_{n} \equiv \hat{P}_{n}^{\prime \prime \prime \prime}=\sum_{m} \hat{P}_{m} G_{m n} .
\end{aligned}
$$

From Eqs. (5.20) and (5.23), we see that

$$
\int \hat{P}_{n} d \rho=\sum_{m} C_{m n} \int P_{m} d \rho=C_{0 n},
$$

and from Eqs. (6.3), likewise,

$$
\begin{array}{ll}
C_{0 n}^{\prime}=\int \hat{P}_{n}^{\prime} d \rho=D_{0 n} ; & C_{0 n}^{\prime \prime}=E_{0 n} ; \\
C_{0 n}^{\prime \prime \prime}=F_{0 n} ; & C_{0 n}^{\prime \prime \prime \prime}=G_{0 n} .
\end{array}
$$

We now take the derivatives of Eq. (5.24), set $\lambda=0$, and use Eqs. (5.25), (6.1), (6.2), (6.5), and the fact that $C_{0 n}(\lambda=0)=\delta_{0 n}$, to obtain

$$
\begin{aligned}
& \left\langle\omega^{2}\right\rangle-1=\left(s \frac{\langle\rho\rangle}{\langle\Lambda\rangle}\right)^{-2}\left[\left(2 E_{00}+2 \sum_{n} e^{-s \hat{\Lambda}_{n} /\langle\Lambda\rangle} D_{0 n}^{2}\right)+\frac{s\left(-\hat{\Lambda}_{0}^{\prime \prime}\right)}{\langle\Lambda\rangle}\right]_{\lambda=0}, \\
& \left\langle\omega^{3}\right\rangle-1=\left(s \frac{\langle\rho\rangle}{\langle\Lambda\rangle}\right)^{-3}\left[\left(-2 F_{00}-6 \sum_{n} e^{-s \hat{\Lambda}_{n} /\langle\Lambda\rangle} E_{0 n} D_{0 n}\right)+\frac{s^{2}\left(-3 \hat{\Lambda}_{0}^{\prime} \hat{\Lambda}_{0}^{\prime \prime}\right)}{\langle\Lambda\rangle^{2}}\right]_{\lambda=0} \\
& +s\left(6 E_{00} \hat{\Lambda}_{0}^{\prime}+\hat{\Lambda}_{0}^{\prime \prime \prime}+6 \sum_{n} e^{-s \hat{\Lambda}_{n} /\langle\Lambda\rangle} D_{0 n}^{2} \hat{\Lambda}_{n}^{\prime}\right) /\langle\Lambda\rangle_{\lambda=0} \\
& \left\langle\omega^{4}\right\rangle-1=\left(s \frac{\langle\rho\rangle}{\langle\Lambda\rangle}\right)^{-4}\left[\left(2 G_{00}+\sum_{n} e^{-s \hat{\Lambda}_{n} /\langle\Lambda\rangle}\left(8 F_{0 n} D_{0 n}+6 E_{0 n}^{2}\right)\right)\right. \\
& +s\left(-8 F_{00} \hat{\Lambda}_{0}^{\prime}-12 E_{00} \hat{\Lambda}_{0}^{\prime \prime}-\hat{\Lambda}_{0}^{\prime \prime \prime \prime}-\sum_{n} e^{-s \hat{\Lambda}_{n} /\langle\Lambda\rangle}\left(24 D_{0 n} E_{0 n} \hat{\Lambda}_{n}^{\prime}+12 D_{0 n}^{2} \hat{\Lambda}_{n}^{\prime \prime}\right)\right) /\langle\Lambda\rangle \\
& \left.+s^{2}\left(4 \hat{\Lambda}_{0}^{\prime} \hat{\Lambda}_{0}^{\prime \prime \prime}+3\left(\hat{\Lambda}_{0}^{\prime \prime}\right)^{2}+12 E_{00}\left(\hat{\Lambda}_{0}^{\prime}\right)^{2}+12 \sum_{n} e^{-s \hat{\Lambda}_{n} /\langle\Lambda\rangle}\left(D_{0 n}\right)^{2}\left(\hat{\Lambda}_{n}^{\prime}\right)^{2}\right) /\langle\Lambda\rangle^{2}+\frac{s^{3}\left[-6\left(\hat{\Lambda}_{0}^{\prime}\right)^{2} \hat{\Lambda}_{0}^{\prime \prime}\right]}{\langle\Lambda\rangle^{3}}\right]_{\lambda=0} .
\end{aligned}
$$

The dependence on $\langle\Lambda\rangle$ in Eqs. (6.6) is illusory, since Eq. (5.32) suggests that $\langle\Lambda\rangle$ can be eliminated entirely. Equations $(6.3)$ and $(6.6)$ remain valid with $\langle\Lambda\rangle$ set equal to 1 if all derivatives in both sets of equations are reinterpreted as derivatives with respect to $\mu=\lambda /\langle\Lambda\rangle$.

It is seen from Eq. (6.6) that the residual moments $\left(\left\langle\omega^{n}\right\rangle-1\right)$, as expected, approach constants for small $s$, and are proportional to $1 / s$ for large $s$.

In Appendix B we calculate $\hat{\Lambda}_{n}^{\prime}, \hat{\Lambda}_{n}^{\prime \prime}, \hat{\Lambda}_{n}^{\prime \prime \prime}, \hat{\Lambda}_{n}^{\prime \prime \prime \prime}$ and $D_{m n}, E_{m n}, F_{m n}, G_{m n}$ from Eqs. (5.14) in terms of $\Lambda_{m}$ and $\rho_{i j}$.

The second, third, and fourth moments of $V(\omega)$ were computed by this procedure, and are com- pared in Figs. 6-8 to the second, third, and fourth factorial moments of the photocount distribution $p(m, T)$ measured by Jakeman, Oliver, Pike, Lax, and Zwanziger. ${ }^{40}$

VII. ASYMPTOTIC APPROXIMATIONS FOR $V(\omega)$ AND $p(m, T)$

For long times ( $s \gg 1)$, Eq. (5.34) is well approximated by its first term alone,

$$
\bar{M}(\nu) \approx e^{-\hat{E}_{0}(\mu) s}\left[C_{00}(\mu)\right]^{2} .
$$

The inverse of Eq. (5.29),

$$
V(\omega)=(1 / 2 \pi i) \int_{a-i \infty}^{a+i \infty} e^{\nu \omega} \bar{M}(\nu) d \nu,
$$

can be written, changing variables according to Eq. (5.33) and inserting Eq. (7.1), 


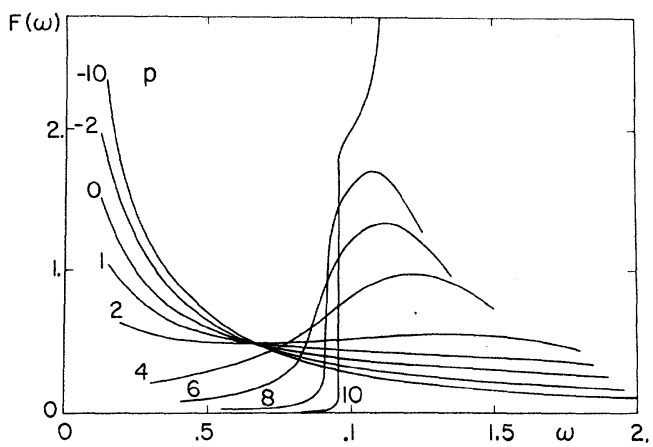

FIG. 13. Functions $F(\omega)$ appropriate to the asymptotic approximation $(s \gg 1)$ of $V(\omega), V(\omega)=s^{1 / 2} F(\omega) e^{-s E}(\omega)$.

$$
V(\omega)=(\langle\rho\rangle s / 2 \pi i) \int e^{-s\left[\hat{E}_{0}(\mu)-\mu\langle\rho\rangle \omega\right]}\left[C_{00}(\mu)\right]^{2} d \mu .
$$

Large $s$ makes the exponential a rapidly varying function compared to $C_{00}^{2}$, and we may integrate Eq. (7.3) by the method of steepest descent. ${ }^{64}$ The point $\mu_{m}$ at which the exponential in Eq. (7.3) is a maximum is an implicit function of $\omega$ :

$$
\omega=\frac{1}{\langle\rho\rangle}\left(\frac{d}{d \mu} \hat{E}_{0}\right)_{\mu_{m}} \Rightarrow \mu_{m}=\mu_{m}(\omega)
$$

We have

$$
\begin{aligned}
V(\omega) \approx & {\left[C_{00}\left(\mu_{m}\right)\right]^{2}\langle\rho\rangle s \exp \left\{-s\left[\hat{E}_{0}\left(\mu_{m}\right)-\mu_{m}\langle\rho\rangle \omega\right]\right\} } \\
& \times \frac{1}{2 \pi i} \int \exp \left[-\frac{1}{2} s \hat{E}_{0}^{\prime \prime}\left(\mu_{m}\right)\left(\mu-\mu_{m}\right)^{2}\right] \cdot(7.5)
\end{aligned}
$$

Replacing $\left(\mu-\mu_{m}\right)$ by $i x$ and integrating, we find

$$
V(\omega)=s^{1 / 2} F(\omega) e^{-s E(\omega)},
$$

where

$$
\begin{aligned}
& F(\omega)=\left[\langle\rho\rangle /(2 \pi)^{1 / 2}\right]\left[C_{00}\left(\mu_{m}\right)\right]^{2} /\left[-\hat{E}_{0}^{\prime \prime}\left(\mu_{m}\right)\right], \\
& E(\omega)=\hat{E}_{0}\left(\mu_{m}\right)-\mu_{m}\langle\rho\rangle \omega .
\end{aligned}
$$

The derivatives of $\hat{E}_{0}$ and $C_{00}$ with respect to $\mu$ are related to the derivatives of $\hat{\Lambda}_{0}$ and $C_{00}$ with respect to $\lambda$. The latter can be computed for $\Lambda_{i}$ and as shown in Appendix B and in Eq. (6.5).

The functions $F(\omega)$ and $E(\omega)$ computed for our laser model are shown in Figs. 13 and 14.

Next, an asymptotic approximation for $p(m, T)$ in the limit of large $\langle m\rangle$ is obtained. Equation (2.8) can be written

$$
p(m, T)=\int d \omega P_{m}(\omega) V(\omega) /\langle m\rangle,
$$

where the normalized Poisson distribution

$$
P_{m}(\omega)=(\langle m\rangle \omega)^{m} e^{-\langle m\rangle \omega}\langle m\rangle / m \text { ! }
$$

has moments

$$
\begin{aligned}
& \langle\omega\rangle_{P}=(m+1) /\langle m\rangle, \\
& \left\langle\omega^{2}\right\rangle_{P}=(m+2)(m+1) /\langle m\rangle^{2} .
\end{aligned}
$$

The variance of $P_{m}(\omega)$,

$$
\left\langle(\Delta \omega)^{2}\right\rangle=\left\langle\omega^{2}\right\rangle_{P}-\langle\omega\rangle_{P}^{2}=(m+1) /\langle m\rangle^{2},
$$

tends to zero for large $\langle m\rangle$ while the variance of $V(\omega)$ tends to zero for large $s$. For a given $s$, $\langle m\rangle$ can be made sufficiently large so that $P_{m}(\omega)$ is sharp compared to $V(\omega)$, and Eq. (7.9) can be integrated to give

$$
p(m, T) \approx \frac{V(m /\langle m\rangle)}{\langle m\rangle} .
$$

For times $T$ short compared to $T_{c}=\langle\Lambda\rangle^{-1}$, the correlation time of intensity fluctuations $\rho$ can be assumed to remain constant under the integral in Eq. (5.3):

$$
\int_{0}^{T} \rho(t) d t \approx \rho T \Rightarrow \omega=\rho /\langle\rho\rangle .
$$

Since the distribution of $\rho$ is the steady state $P_{0}$ defined by Eqs. (5.9) and (5.19),

$$
P_{0}(\rho)=N e^{p \rho / 2-\rho^{2} / 4},
$$

where $N$ is a normalization factor, we have, in this limit,

$$
V(\omega)=N \exp \left[\frac{1}{2} \omega p\langle\rho\rangle-\left(\frac{1}{2} \omega\langle\rho\rangle\right)^{2}\right], \quad s \ll 1 .
$$

The moments of $V(\omega)$, according to Eq. (7.15), are $\left\langle\omega^{r}\right\rangle=\left\langle\rho^{r}\right\rangle /\langle\rho\rangle^{r}=\left(N /\langle\rho\rangle^{r}\right) \int_{0}^{\infty} \rho^{r} \exp \left(\frac{1}{2} p \rho-\frac{1}{4} \rho^{2}\right) d \rho$,

and can be calculated from the following formula, obtained from Eq. (7.18) or integrating by parts (see Ref. 15, footnote 27):

$$
\left\langle\rho^{r}\right\rangle=p\left\langle\rho^{r-1}\right\rangle+2(r-1)\left\langle\rho^{r-2}\right\rangle \text {. }
$$

In particular,

$$
\begin{aligned}
& \langle\rho\rangle=p+\sqrt{2}\left(e^{p^{2} / 4} \int_{-p / \sqrt{2}}^{\infty} e^{-t^{2} / 2} d t\right)^{-1}, \\
& \quad+ \\
& \left\langle\rho^{2}\right\rangle=p\langle\rho\rangle+2 .
\end{aligned}
$$

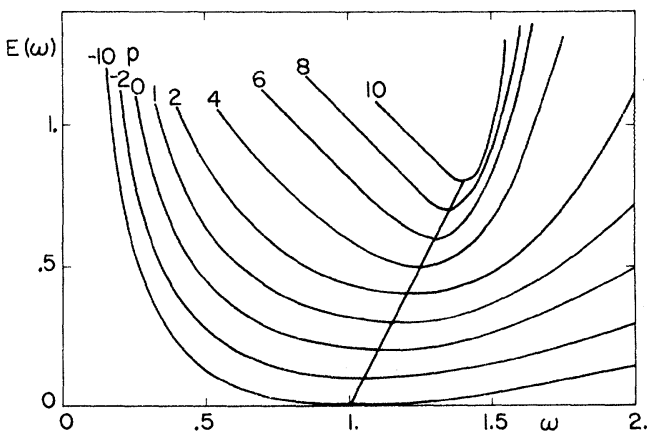

FIG. 14. Functions $E(\omega)$ appropriate to the asymptotic approximation $(s \gg 1)$ of $V(\omega), V(\omega)=s^{1 / 2} F(\omega) e^{-s E(\omega)}$. For all curves, $E(\omega=1)=0$; they are displaced for clarity by the length of the line connecting the minima to the point $\omega=1, E=0$. 


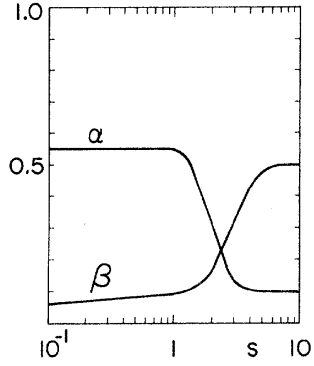

FIG. 15. Scale factors $\alpha$ and $\beta$ used in computations described in Sec. VIII.

\section{INVERSE LAPLACE TRANSFORM:} DISCRETE APPROXIMATIONS

There is no known general procedure of numerically inverting a Laplace transform, known only on the real axis, guaranteed to give accurate results in every case. The difficulties involved are well illustrated in the book by Bellman, Kalaba, and Lockett, ${ }^{65}$ and they all arise from the fact that the Laplace transform is a smoothing operation. Significant figures will be lost on the inverse unsmoothing operation. Each problem must be tackled individually and the only useful guide is an a priori knowledge of the general nature of the solution. In our case, we know that $V(\omega)$ are normalized positive functions, zero at the origin and approaching zero for large values of $\omega$.

The over-all shapes of $V(\omega)$ were first studied by obtaining discrete sets of values $V\left(\omega_{i}\right)$ as functions of $p$ and $s$. An extensive collection of possible ways is given in the book by Krylov and Skoblya. ${ }^{66}$ For example, Eq. (5.29) can be written, with $\omega=\alpha x$,

$$
\bar{M}(\nu)=\int_{0}^{\infty} e^{-x}\left[\alpha e^{x} e^{-\nu \alpha x} V(\alpha x)\right] d x .
$$

By use of the Gauss-Laguerre integration formula, ${ }^{67}$

$$
\int_{0}^{-\infty} e^{-x} f(x) d x \approx \sum_{i=1}^{n} A_{i} f\left(x_{i}\right),
$$

one approximates Eq. (8.1) as

$$
\bar{M}(\nu) \approx \sum_{i=1}^{n} \alpha A_{i} e^{(1-\alpha \nu) x_{i}} V\left(\alpha x_{i}\right) .
$$

The choice of a linear set of points $\nu_{j}=\beta j, j=0,1$, $\ldots, n-1$ (which is not the only, nor necessarily the optimum, choice), gives a system of algebraic equations

$$
\bar{M}_{j}=\sum_{i=1}^{n} G_{i j} V_{i}
$$

with

$$
V_{i}=V\left(\omega_{i}\right)=V\left(\alpha x_{i}\right),
$$

$$
\bar{M}_{\tilde{j}}=\bar{M}(\beta j), \quad G_{i j}=\alpha A_{i} e^{1-\alpha \beta \bar{j}} .
$$

The nature of the problem makes $G_{i j}$ an ill-conditioned $^{68}$ matrix, that is, significant digits are lost in the calculation of its inverse. One tries to mitigate this detrimental effect by experimenting with different quadrature formulas (which yield different sets of $x_{i}$ and $A_{i}$ ) or values of the scale factors $\alpha$ and $\beta$.

The 15-point Gauss-Laguerre integration formula was found to work well over our entire - 10 $\leq p \leq 10$ range. The values of $A_{i}$ and $x_{i}$ were obtained from Ref. 69 where $A_{i}$ are denoted $\alpha_{i}$. The optimum parameters $\alpha$ and $\beta$, found by trial and error, varied with $s$, but were independent of $p$. Figure 15 summarizes their behavior. Figure 16 shows typical results, with good and poor choices of $\alpha$ and $\beta$, compared to continuous approximations of $V(\omega)$ obtained as described in Secs. IX and X.

The $\bar{M}_{j}$ defined by Eq. (8.5) were computed using the matrix method of Eqs. (5.32) and (5.34).

\section{INVERSE LAPLACE TRANSFORM: CONTINUUOUS APPROXIMATIONS}

To obtain continuous functions $V(\omega)$ rather than discrete sets of values, we introduce the new procedure of representing $V(\omega)$ by a judiciously chosen function times $a$ polynomial. The $N$-polynomial coefficients are determined to satisfy $N$ values of the Laplace transform. The choice of the approximating function is guided by the information we already have on $V(\omega)$.

In the region $-10 \leq p \leq 1$ we represent $V(\omega)$ by

$$
V(\omega)=[\Gamma(a)]^{-1} a^{a} \omega^{a-1} e^{-a \omega} \sum_{n=0}^{N} b_{n} \omega^{n}
$$

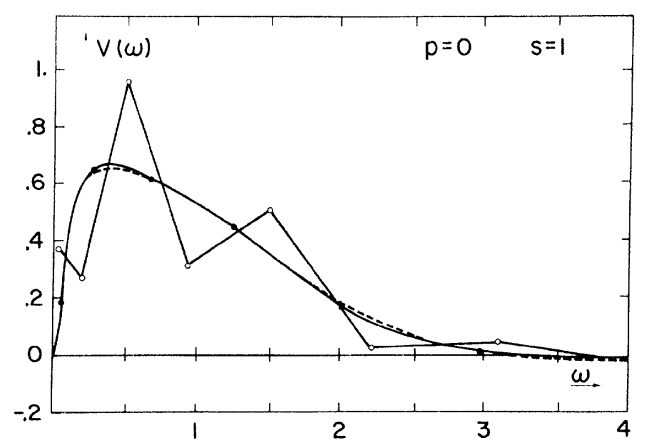

FIG. 16. Comparison of $V(\omega)$ distributions computed numerically by inverting a Laplace transform with different methods. The solid smooth curve is obtained by the representation method, Eq. (9.1) of the text. The dashed curve is obtained by the Fourier-transform method of Sec. $\mathrm{X}$; where not apparent, it coincides with the previous curve. The solid circles are the result of the "discrete" technique of Sec. VIII; the open circles, connected by straight lines for clarity, exemplify a poor choice of scaling parameters $\alpha$ and $\beta$ in the discrete technique. 
We call the function multiplying the polynomial in Eq. (9.1) the Rice-Mande $1^{70}$ approximation. It is normalized and has unit first moment, and $a$ is chosen so that it approximates the correct second moment of $V(\omega)$ known from Eq. (6.6a) as a function of $s$ and p. From Eqs. (5.29) and (9.1), one obtains

$$
\bar{M}(\nu)=\frac{a^{a}}{\Gamma(a)} \sum_{n=0}^{N} b_{n} \frac{\Gamma(a+n)}{(\nu+a)^{a+n}},
$$

and the moments of $V(\omega)$, defined by Eq. (9.1), are

$$
\left\langle\omega^{r}\right\rangle=\sum_{n} b_{n} \frac{\Gamma(a+n+r)}{\Gamma(a) a^{n+r}} .
$$

In the region $1<p \leq 10$, we use the approximations

$$
V(\omega)=\bar{N} e^{\alpha \omega-(\beta \omega / 2)^{2}} \sum_{n=0}^{N} d_{n} \omega^{n}
$$

and

$$
\bar{M}(\nu)=\frac{\bar{N} e^{y^{2}}}{\beta} \sum_{n} d_{n}\left(\frac{2}{\beta}\right)^{n} \int_{-y}^{\omega}(x+y)^{n} e^{-x^{2}} 2 d x,
$$

where

$$
x=\frac{1}{2} \omega \beta-\alpha / \beta, \quad y=(\alpha-\nu) / \beta,
$$

and $\bar{N}=\bar{N}(\alpha / \beta)$ is the normalization factor of the Gaussian (truncated at $\omega=0$ ) multiplying the polynomial. Again, $\alpha$ and $\beta$ are chosen so that the Gaussian approximates the first and second moments of $V(\omega)$ as $s$ and $p$ vary. The moments of Eq. (9.4) are given by

$$
\left\langle\omega^{r}\right\rangle=\bar{N} e^{(\alpha / \beta)^{2}} \sum_{n} d_{n}\left(\frac{2}{\beta}\right)^{n+r} \int_{-\alpha / \beta}^{\infty} x^{n+r} e^{-x^{2}} 2 d x,
$$

where the integrals can be written in terms of the incomplete $\Gamma$ function. Equation (9.7) can be reduced by partial integration to

$$
\left\langle\omega^{r}\right\rangle=\sum_{n} d_{n} I_{n+r},
$$

where

$$
I_{m}=\left(2 \alpha / \beta^{2}\right) I_{m-1}+\left(2 / \beta^{2}\right)(m-1) I_{m-2},
$$

with

$$
\begin{aligned}
& I_{0}=1, \quad I_{1}=2 \alpha / \beta^{2}+(2 / \beta) E, \\
& E^{-1}=e^{(\alpha / \beta)^{2}} \int_{-\alpha / \beta}^{\infty} e^{-x^{2}} 2 d x .
\end{aligned}
$$

We use linear sets of points $\nu_{j}=\gamma j, j=0,1, \ldots$, $N-1$ for determining $b_{n}$ and $d_{n}$ by Eqs. (9.2) and (9.5). The algebraic system obtained from Eq. (9.2) was found to be least ill conditioned when $\gamma$ $=0.1$ for all choices of $s$ and $p$. For the system obtained from Eq. (9.5) it was necessary to vary $\gamma$ from 0.2 to 1.0 as $\left[\left\langle\omega^{2}\right\rangle^{-1}\right]$ varied from about 0.3 to about 0.0003 . Up to 15 coefficients were necessary for expansion (9.2) when $V(\omega)$ displayed a sharp rise near the origin (small values of $s$ and $\omega)$.

For the coefficients $b_{n}$ and $d_{n}$ for $p=-10,-2$, $-1,0,1,2,4,6,8,10$ and $s=0.1,0.2,0.5,1$, $2,5,10,20,50$, and 100 see Ref. 47. Figures 10-12 show some of the $V(\omega)$ curves obtained by Eqs. (9.1) and (9.4). Although we did not compute $V(\omega)$ for $p$ less than -10 or more than 10 , the representations used in Eqs. (9.1) and (9.4) are definitely suitable in those ranges as well for reasons to be discussed below.

For $p<-10$, the laser statistics are already close to those of a damped harmonic oscillator as is evident in Fig. 17. (The exact results for the harmonic oscillator are obtained in Sec. X.) Figure 17 also illustrates the breakdown, at small $s$, of the Rice-Mandel approximation [see comment after Eq. (9.1)],

$$
\begin{aligned}
& V(\omega)_{\mathrm{RM}}=[\Gamma(a)]^{-1} a^{a} \omega^{a-1} e^{-a \omega}, \\
& a^{-1}=\left\langle\omega^{2}\right\rangle-1,
\end{aligned}
$$

for the laser below threshold (and thermal fields). The Rice-Mandel curve shown has the same second moment as the RWVP curve shown.

For $p>10$, the laser is already amplitude stabilized $\left(\left\langle(\Delta \rho)^{2}\right\rangle /\langle\rho\rangle^{2}\right.$ is small, as shown in Fig. 1), permitting one to make the usual quasilinear approximation. $^{71}$ By expanding the drift and diffusion coefficients of Eq. (5.19) in series about their mean value, and by retaining linear terms only, Eq. (5.19) is reduced to

$$
L \approx-\frac{\partial}{\partial \rho}(A+B \rho)-C \frac{\partial^{2}}{\partial \rho^{2}},
$$

where $A, B$, and $C$ are independent of $\rho$ but functions of $p$. This results in a Gaussian distribution for $\rho$, which in turn implies a Gaussian distribution for $\omega$ :

$$
\begin{aligned}
V(\omega)_{\mathrm{QL}}=[2 & \left.\left(\left\langle\omega^{2}\right\rangle-1\right)\right]^{-1 / 2} \\
& \times \exp \left[-\frac{1}{2}(\omega-1)^{2} /\left(\left\langle\omega^{2}\right\rangle-1\right)\right] .
\end{aligned}
$$

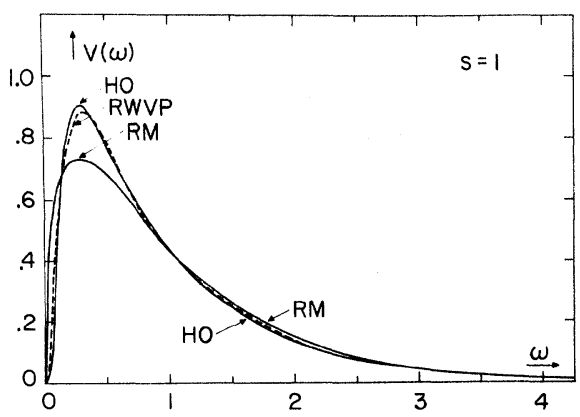

FIG. 17. Comparison of $V(\omega)$ distributions given by the harmonic-oscillator (HO) model of a laser operating below threshold, by the RWVP oscillator model at $p=-10$, and by the Rice-Mandel (RM) approximation determined by the second moment of the HO curve. 


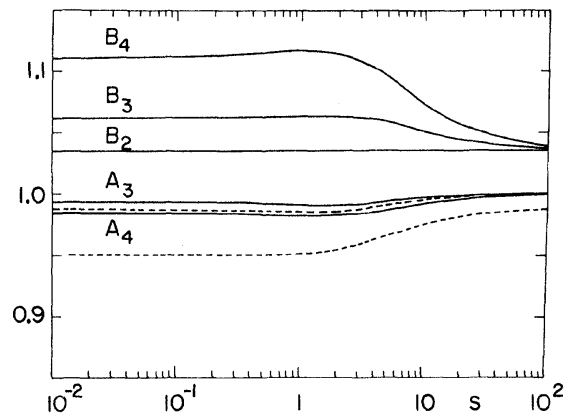

FIG. 18. Comparison of moments of $V(\omega)$ obtained from the RWVP model and from models of the laser operating well above and well below threshold. $B_{n}$ $=\left(\left\langle\omega^{n}\right\rangle-1\right)_{\mathrm{HO}} /\left(\left\langle\omega^{n}\right\rangle-1\right)_{\mathrm{RWVP}}$, where HO stands for the damped harmonic-oscillator model, and $p=-10$ was used in the RWVP model. $A_{n}=\left(\left\langle\omega^{n}\right\rangle-1\right)_{\mathrm{RWVP}} /\left(\left\langle\omega^{n}\right\rangle-1\right)_{\mathrm{QL}, \mathrm{GL}}$, where QL stands for the quasilinear model (solid lines) and GL stands for the Glauber-Lachs (Refs. 72 and 73) model (dashed lines) of a superposition of coherent and incoherent fields. $A_{2}$ was set equal to unity, and $p=10$ was used in the RWVP model.

[Since $V(\omega)_{Q \mathrm{~L}}$ is highly localized around $\omega=1$, we integrate over $-\infty<\omega<\infty$ to obtain the normalization factor. ] The higher moments of $V(\omega)_{\mathrm{QL}}$ of Eq. (9.15) are determined by the second moment:

$\left\langle\omega^{3}\right\rangle_{Q L}-1=3\left(\left\langle\omega^{2}\right\rangle_{Q L}-1\right)$,

$\left\langle\omega^{4}\right\rangle_{\mathrm{QL}}-1=6\left(\left\langle\omega^{2}\right\rangle_{\mathrm{QL}}-1\right)+3\left(\left\langle\omega^{2}\right\rangle_{\mathrm{QL}}-1\right)^{2}$.

In Fig. 18, we display ratios of the moments of the harmonic-oscillator model to the moments of the RWVP model at $p=-10$, and ratios of the moments of the RWVP model at $p=10$ to the moments of the quasilinear model, using the second moment of the RWVP model at $p=10$. The proximity to unity of the various ratios is a good indication that we covered the full range of $p$ for the RWVP model of the laser which cannot be handled by simpler methods.

It may be of interest to note that an early model proposed by Glauber ${ }^{72}$ and Lachs ${ }^{73}$ for a laser above threshold is a superposition of a perfect coherent signal with a Gaussian noise. Jakeman and Pike ${ }^{74}$ and Jaiswal and Mehta ${ }^{75}$ calculated the moments of $V(\omega)_{\mathrm{GL}}$ [i.e., the factorial moments of $p(m, T)]$ for such a field, assuming a Lorentzian spectrum. Choosing the ratio of coherent to incoherent signals so as to match the second moment of $V(\omega)$ for the RWVP model at $p=10$, we calculated the corresponding third and fourth moments of $V(\omega)_{\mathrm{GL}}$ for the Glauber-Lachs model. The ratios of the moments of the RWVP model at $p=10$ to the moments of the Glauber-Lachs model are shown as dashed curves in Fig. 18. By comparing these with similar curves for the quasilinear model it is safe to conclude that the quasilinear model is a better approximation to the laser above threshold than the Glauber-Lachs model.

\section{ACCURACY OF COMPUTATIONS}

Three independent tests were performed to check the accuracy of our continuous representation method for obtaining the inverse Laplace transform $V(\omega)$.

\section{A. Laser below Threshold}

The operator

$$
L=\frac{\partial}{\partial \rho}(2 p \rho+4)-\frac{\partial^{2}}{\partial \rho^{2}}(4 \rho),
$$

which is Eq. (5.19) without the $\rho^{2}$ term, has eigenfunctions and eigenvalues

$$
\begin{aligned}
& P_{n}(\rho)=\frac{1}{2}|p|(1 / n !) e^{-|p| \rho / 2} L_{n}\left(\frac{1}{2}|p| \rho\right), \\
& \Lambda_{n}=2|p| n,
\end{aligned}
$$

where $L_{n}$ are the Laguerre polynomials, and $|p|$ stands for $-p$ since $p<0$. The matrix elements defined by Eq. (5.22) are

$$
\begin{aligned}
& \rho_{i i}=(2 /|p|)(2 i+1), \\
& \rho_{i, i+1}=\rho_{i+1, i}=-(2 /|p|)(i+1),
\end{aligned}
$$

all others zero. The effective linewidth $\langle\Lambda\rangle$ is calculated to be

$$
\langle\Lambda\rangle=\Lambda_{1}=2|p| \text {. }
$$

Equation (10.1) corresponds to a damped harmonic-oscillator model of a laser operating below threshold, whose field $\beta$ is described (see Ref. $25)$ by the Langevin equations:

$$
\begin{aligned}
& \frac{d x}{d t}=-|p| x+F_{x}(t), \\
& \frac{d y}{d t}=-|p| y+F_{y}(t), \\
& \beta=x-i y,
\end{aligned}
$$

where $|p|$ is a dissipation coefficient and $F_{x}$ and $F_{y}$ are random Markoffian forces which account for the interaction with reservoirs. Equation (10.1) can be obtained similarly to the way Eq. (3.4) of Ref. 15 is calculated from Eqs. (2.1) and (2.2) of that reference.

$M\left(\lambda^{\prime}\right)$, defined by our Eqs. (5.1) and (5.2), is given by Eq. (18C31) of Ref. 25,

$$
M\left(\lambda^{\prime}\right)=e^{s^{\prime}}\left[\cosh z s^{\prime}+\frac{1}{2}(z+1 / z) \sinh z s^{\prime}\right]^{-1},(10.7)
$$

where

$$
s^{\prime}=|p| T, \quad z=\left(1+4 \lambda^{\prime} \epsilon\langle\rho\rangle /\langle\Lambda\rangle\right)^{1 / 2} .
$$

The inverse Laplace transform of Eq. (10.7),

$$
W(\Omega)=(1 / 2 \pi i) \int_{C} e^{\lambda \cdot \Omega} M\left(\lambda^{\prime}\right) d \lambda^{\prime},
$$

can be integrated by the usual residue methods 


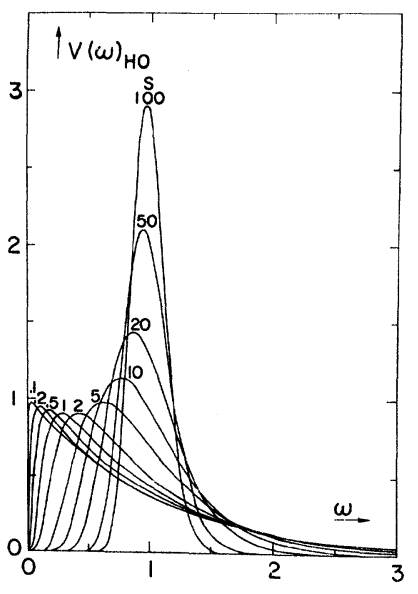

FIG. 19. Probability distribution $V(\omega)$ of the normalized $(\langle\omega\rangle=1)$ time-integrated light intensity $\omega$ computed from the damped harmonic-oscillator model of a laser operating well below threshold (thermal source) for various values of the scaled time $s$.

giving, after scaling the result according to Eqs. (5.3) and (5.4),

$$
\begin{aligned}
V(\omega)= & 2 s^{\prime} \sum_{n} y_{n}^{2} \exp \left\{\left[1-\frac{1}{2}\left(y_{n}^{2}+1\right) \omega\right] s^{\prime}\right\} \\
& \times\left[\left(2 y_{n} s^{\prime}+y_{n}+1 / y_{n}\right) \sin y_{n} s^{\prime}\right. \\
& \left.+s^{\prime}\left(y_{n}^{2}-1\right) \cos y_{n} s^{\prime}\right]^{-1},
\end{aligned}
$$

where $y_{n}$ are the positive roots of the equation

$$
\tan y s^{\prime}=2 y /\left(y^{2}-1\right) \text {. }
$$

This result was published earlier by Jakeman and Pike. ${ }^{76}$ Equations (10.8), (10.4), and (5.25) define the relation

$$
s^{\prime}=\frac{1}{2}\langle\Lambda\rangle T=\frac{1}{2} s
$$

between the Jakeman-Pike time parameter $\left(s^{\prime}\right)$ and ours $(s)$. It is also to be noted that $\frac{1}{2}\langle\Lambda\rangle$ is approximately equal to the half-width at half-power of the total (phase and amplitude) noise spectrum only for the laser below threshold (see Ref. 15). Above threshold this is not true.

We calculated $V(\omega)$ for a set of values of $s$ using the residue method of Eq. (10.10) (see Fig. 19). The same distributions were also calculated using the representation method, Eq. (9.1) with $\bar{M}(\nu)$ computed from Eqs. (5.31), (5.34), and (10.2)(10.4). Agreement to two significant figures was generally found, which bolstered our confidence in the representation technique.

\section{B. Reduction of the Inverse Laplace Transform to a Fourier} Series

Equation (7.2) can be converted to a Fourier transform by the change of variables $\nu=a+i x$ :

$$
V(\omega)=\left(e^{a \omega} / 2 \pi\right) \int_{-\infty}^{\infty} e^{i \omega x} \bar{M}(a+i x) d x .
$$

Since from its very nature $V(\omega)$ approaches zero for large $\omega$, we may assume $V(\omega) e^{-a \omega}$ to be effectively zero outside some interval $\left(0, \omega_{M}\right)$, and expand it in a Fourier series:

$$
V(\omega) e^{-a \omega}=\sum_{-\infty}^{\infty} C_{k} e^{i k \sigma \omega},
$$

where

$$
\begin{aligned}
\sigma & =\left(2 \pi / \omega_{M}\right), \\
C_{k} & =(\sigma / 2 \pi) \int_{0}^{\omega_{M}}\left[V(\omega) e^{-a \omega}\right] e^{-i k \sigma \omega} d \omega \\
& \approx(\sigma / 2 \pi) \int_{0}^{\infty}\left[V(\omega) e^{-a \omega}\right] e^{-i k \sigma \omega} d \omega .
\end{aligned}
$$

The integral in Eq. (10.16) is just the Laplace transform of $V(\omega)$ at $a+i k \sigma$. Comparison with Eq. (5.29) then yields

$$
C_{k}=(\sigma / 2 \pi) \bar{M}(a+i k \sigma) \text {. }
$$

Writing

$$
\bar{M}(a+i k \sigma)=A_{k}+i B_{k},
$$

Eq. (10.14) becomes

$V(\omega)=e^{a \omega} \frac{\sigma}{2 \pi}\left(A(0)+2 \sum_{k=1}^{\infty}\left(A_{k} \cos k \sigma \omega-B_{k} \sin k \sigma \omega\right)\right)$.

The use of Eq. (10.19) requires the calculation of $M$ for large complex arguments, and the finite matrix procedure of Eq. (5.31) may no longer be adequate. We used the Numerov method ${ }^{77,78}$ for solving the eigenvalue problem of Eq. (5.14) and calculated $\bar{M}$ using Eq. (5.34). Owing to the slow convergence of the Fourier series, Eq. (10.19), about ten times more computation time is required to obtain one $V(\omega)$ curve, as compared to the representation method based on real values of $\lambda$. We limited ourselves to the computation of a few test cases. First, we computed $V(\omega)$ for the damped harmonic-oscillator model using the operator of Eq. (10.1) and the parameters $s=1, a=0, \sigma=1, k$ $=1,2, \ldots, 40$. The result agrees within $1 \%$ of peak value with the distribution calculated from Eq. (10.10), and would be indistinguishable from the curve displayed in Fig. 17. We also computed $V(\omega)$ for the RWVP at $p=-10$, with the same parameters as above, again obtaining excellent agreement with the RWVP curve displayed in Fig. 17 , computed by the representation method. Finally, we computed $V(\omega)$ for the RWVP model at $p=0$ using the same parameters as above. The result is shown in Fig. 16, along with results obtained by other methods for comparison. The small discrepancy between the representation method and the Fourier-series method is not necessarily an error of the representation method. The Fourier method was used with 20 points as well as with the 40 points on which Fig. 16 is 


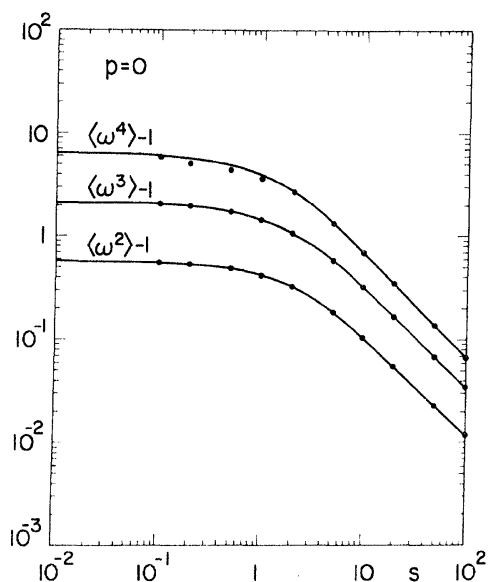

FIG. 20. Moments of $V(\omega)$ for $p=0$ (threshold). The solid lines are obtained by the exact perturbation method of Sec. VI. The dots correspond to the continuous representation described in Sec. IX, and were computed from the data contained in Table V (Ref. 47); $\left\langle\omega^{r}\right\rangle=\left\langle m^{[r]}\right\rangle /\langle\omega\rangle^{r}$.

based. Improved agreement accompanied the change from 20 to 40 points. It might take 200 points to be sure of convergence of the Fourier method. Although the Fourier-series method is potentially more accurate, because it avoids illconditioned matrices, we thought it too expensive to generate families of $V(\omega)$ curves.

\section{Comparison of Moments of $V(\omega)$}

Moments of $V(\omega)$ were calculated using Eqs. (9.3) and (9.8). These are based on our representation method of inverting the Laplace transform, and are shown as points in Fig. 20. The points agree quite well with the curves obtained from the potentially exact formulas (6.1) based on perturbation theory.

In summary, we believe our $V(\omega)$ curves are generally accurate to about $1 \%$ of peak value except when the $V(\omega)$ curves rise sharply near the origin (small $s$ and small $\omega$ ). The reason for this confidence is based on (a) agreement between representation results with exact residue results for the harmonic-oscillator model, (b) agreement of representation method (real $\lambda$ information) with the "exact" Fourier method (inversion of the Laplace transform in the complex plane), (c) agreement of representation moments with "exact" moments, (d) agreement between the representation method and the asymptotic results of Sec. VII for large $s(s \geq 10)$.

\section{DISCUSSION AND COMPARISON WITH EXPERIMENT}

The problem of determining the statistical dis tribution of the integrated intensity of a Gaussian random variable has been given considerable at- tention in the past, ${ }^{1}$ mostly because of its relation to the study of noise in amplifier systems like laser sources. We have shown (see, for example, Fig. 17) that the light field of a laser operating in a single mode below threshold behaves like a thermal light field, i. e., it is described by a random complex variable with a Gaussian probability distribution and Lorentzian frequency spectrum. Well above threshold, on the other hand, laser light is ade quately described by a quasilinear approximation which leads to a Gaussian distribution in intensity and a single effective decay rate. Because a superposition of a coherent field with Gaussian noise was thought to be a good approximation to a laser well above threshold, an extensive literature was built up on photocount statistics for such a model. Although we have shown that the quasilinear approximation is more accurate (see Fig. 18) and easier to apply, we have, nevertheless, reproduced and extended a table of Jakeman and Pike ${ }^{2}$ that summarizes the initiating papers in this theoretical literature (see Table $\Pi$ ). This literature is still relevant for understanding scattering and heterodyning experiments that mix coherent and Gaussian signals. A similar bibliography to the experimental photocount statistics of laser and thermal light is given in Table III. Near threshold, the nonlinearities in the laser equations must be retained, and calculating the time-dependent photocount statistics of the field is a complicated quantum-mechanical problem. We made use of an exact quantum classical correspondence, valid for Markoffian processes, to associate our operator variables to classical variables, and then calculated the distribution of the integrated intensity of the field variable from classical equations of motion corresponding to the laser system.

Measurements (see Table III) substantiate the RWVP oscillator model for a laser operating near threshold. Following publication of our main results, ${ }^{38}$ photocount experiments were performed that are in excellent agreement with our theory. In Figs. 3-5, we show the time-dependent photocount distributions measured by Meltzer, Davis, and Mandel, ${ }^{39}$ and in Figs. 6-8, the time-dependent moments measured by Jakeman, Oliver, and Pike $^{40}$ are displayed. Any small discrepancies between theory and experiment are comparable to the quoted errors in theory and experiment.

\section{ACKNOWLEDGMENT}

We would like to thank Dr. John R. Klauder for a careful reading of the manuscript.

\section{APPENDIX A: TIME REVERSAL AND THE EIGENFUNCTIONS OF $L \dagger$}

The purpose of this appendix is to show that the relation $\hat{\varphi}_{n}^{*}(\overrightarrow{\mathrm{a}})=\hat{P}_{n}\left(\overrightarrow{\mathrm{a}}^{T}\right) / P_{0}(\overrightarrow{\mathrm{a}})$ of Eq. $(5.17)$ relating 
TABLE II. Theoretical photocount statistics of Gaussian-Lorentzian and coherent fields, a bibliography of initial papers [after Jakeman and Pike (Ref. 2) with additions]. The numbers in parentheses are the year of publication. The following notation is used: -----, incoherent-field spectrum; $\omega_{c}$, frequency of the coherent field; $\omega_{0}$, mean frequency of the incoherent field; $\langle\Lambda\rangle$, linewidth of the incoherentfield spectrum; $\left\langle I_{c}\right\rangle$, mean intensity of the coherent field; $\left\langle I_{i}\right\rangle$, mean intensity of the incoherent field; (a), mathematically equivalent calculation; (b), not formal expansion in $1 / s, s=\langle\Lambda\rangle T$; (c), in these early papers, bunching was thought to be a property of photons; (d), recurrence relations only; (e), numerical calculations; (f), to second order in $1 / s$; $(\mathrm{g})$, various spectral shapes; $\mathrm{B}$, Bédard (Ref. 3); G, Glauber (Refs. $41,42,72$ ); H, Helstrom (Ref. 84); JM, Jaiswal and Mehta (Ref. 85); JP, Jakeman and Pike (Ref. 86); L, Lachs (Ref. 73); M, Mandel (Ref. 87); MP, McLean and Pike (Ref. 88); P, Pěrina (Ref. 89); PH, Perrina and Horák (Ref. 90); Pu, Purcell (Ref. 91); R, Rice (Ref. 70).

\begin{tabular}{|c|c|c|c|c|c|c|}
\hline FIELD & LIMIT & $M(\lambda)$ & $V(\omega)$ & $p(m, T)$ & $n(2)$ & $n(r)$ \\
\hline & & $\begin{array}{l}J P(69) \\
P H(69) \\
J M(70)\end{array}$ & $\mathrm{PH}(69)$ & $\begin{array}{l}\text { PH (69) } \\
L(71)(g)\end{array}$ & $\begin{array}{l}J P(69) \\
P H(69 \\
J M(70)\end{array}$ & $\begin{array}{l}\mathrm{PH}(70) \\
\mathrm{JM}(70)\end{array}$ \\
\hline & $\omega_{c}=\omega_{0}$ & $\begin{array}{l}J P(69) \\
P H(69) \\
J M(70)\end{array}$ & $\mathrm{PH}(69)$ & $\mathrm{PH}(69)$ & $\begin{array}{l}\operatorname{JP}(69) \\
P H(69) \\
J M(70)\end{array}$ & $\begin{array}{l}\mathrm{PH}(69) \\
\mathrm{JM}(70)\end{array}$ \\
\hline & $\langle\Lambda\rangle \rightarrow 0$ & $\begin{array}{l}\mathrm{JP}(69) \\
\mathrm{PH}(69) \\
\mathrm{JM}(70)\end{array}$ & $\begin{array}{l}\mathrm{JP}(69) \\
\mathrm{PH}(69)\end{array}$ & $\mathrm{PH}(69)$ & $\begin{array}{l}\operatorname{JP}(69) \\
\mathrm{PH}(69) \\
\mathrm{JM}(70)\end{array}$ & $\begin{array}{l}\mathrm{PH}(69) \\
\mathrm{JM}(70)\end{array}$ \\
\hline & $\begin{array}{l}<\Lambda>-\infty \\
\omega_{C}=\omega_{0}\end{array}$ & $\begin{array}{l}J P(69) \\
J M(70)\end{array}$ & $J P(69)$ & $J P(69)$ & $\begin{array}{l}J P(69) \\
J M(70)\end{array}$ & $\begin{array}{l}J P(69) \\
J M(70)\end{array}$ \\
\hline & $\begin{array}{l}\langle\Lambda\rangle \rightarrow 0 \\
\omega_{q}=\omega_{0}\end{array}$ & $G(66)$ & $\begin{array}{l}R(45)(a) \\
P(67)\end{array}$ & $\begin{array}{l}G(66) \\
L(65)\end{array}$ & $L(65)$ & $\begin{array}{l}R(45)(a) \\
G(66) \\
L(65)(d)\end{array}$ \\
\hline & $\left\langle\mathrm{I}_{\mathrm{c}}\right\rangle=0$ & $H(64)$ & $J P(68)(e)$ & $\begin{array}{l}G(65) \\
B(66)(d) \\
J P(68)(e) \\
L(71)(g)\end{array}$ & $\begin{array}{l}B(66)(e) \\
J P(68)\end{array}$ & $B(66)(e)$ \\
\hline & $\begin{array}{l}\left\langle I_{C}\right\rangle=0 \\
\langle\Lambda\rangle \rightarrow 0\end{array}$ & $G(65)$ & $\begin{array}{l}R(45)(a) \\
M(64)\end{array}$ & $M(59)$ & $\begin{array}{l}R(45)(a) \\
M(58)\end{array}$ & $\begin{array}{l}R(45)(d) \\
G(66) \\
L(65)(d)\end{array}$ \\
\hline$\ldots$ & $<\Lambda>-\infty$ & $\begin{array}{l}G(65)(b) \\
H(64)(b)\end{array}$ & $J P(68)$ & $\begin{array}{l}M(59)(b) \\
M P(65) \\
J P(68)(f)\end{array}$ & $\begin{array}{l}\mathrm{Pu}(56)(\mathrm{c}) \\
\mathrm{M}(58)(\mathrm{c}) \\
G(65)\end{array}$ & $G(65)$ \\
\hline & $\left\langle I_{i}\right\rangle=0$ & $G(65)$ & $\begin{array}{l}R(45)(a) \\
M(64)\end{array}$ & $\begin{array}{l}G(63) \\
M(64)\end{array}$ & $\begin{array}{l}R(45)(a) \\
G(65)\end{array}$ & $\begin{array}{l}R(45)(a) \\
G(65)\end{array}$ \\
\hline
\end{tabular}

the eigenfunctions of $L^{\dagger}$ to those of $L$ is a consequence of time reversal and stationarity. The proof given here will generalize an earlier proof (Ref. 25, Chap. 8) from a Fokker-Planck process to a general Markoffian process, and from variables even under time reversal to variables odd under time reversal.

The original version of this paper made the first generalization. Risisen ${ }^{92}$ and Graham and Haken ${ }^{93}$ in the meantime have considered the FokkerPlanck process with odd variables. We shall, therefore, briefly present a proof which makes both generalizations simultaneously.

If the steps in Eq. (8D1-7) of Ref. 25 are repeated without assuming variables even under time reversal, we obtain the condition

$$
P\left(a, t ; a_{0}, t_{0}\right)=P\left(\bar{a}_{0}, t ; \bar{a}, t_{0}\right)
$$

on the two-time probability where $a$ represents a set of variables denoted $\vec{a}$ in the text, and $\bar{a}$ is the set of time-reversed variables, called $\vec{a}^{T}$ in the text. Thus, for any component variable,

$$
\bar{a} \equiv K a K^{-1}= \pm a,
$$

where $K$ is the time-reversal operator which leaves unchanged even variables and reverses the sign of variables odd under time reversal. Condition (A1) has been given a detailed justification by De Groot and Mazur. ${ }^{94}$

Equation (A1) can be rewritten in terms of conditional probabilities,

$$
P\left(a, t \mid a_{0}, t_{0}\right) P_{0}\left(a_{0}\right)=P\left(\bar{a}_{0}, t \mid \bar{a}, t_{0}\right) P_{0}(\bar{a}),
$$

where $P_{0}(a)$ is time independent in the (assumed) stationary case. If the limiting condition

$$
\lim P\left(a, t \mid a_{0}, t_{0}\right)=\delta\left(a-a_{0}\right) \quad \text { as } t \rightarrow t_{0}
$$

is applied to Eq. (A3), one must conclude that the stationary state $P_{0}(a)$ obeys

$$
P_{0}(a)=P_{0}(\bar{a}) \text {. }
$$

When transition probabilities exist, i. e., for $a$ $\neq a^{\prime}$,

$$
\left.\lim P(a, t+\Delta t) \mid a^{\prime}, t\right) / \Delta t=w\left(a, a^{\prime}\right) \text { as } \Delta t \rightarrow 0
$$

exists, and the Chapman-Kolmogoroff equation for a Markoff process can be rewritten as a master equation:

$\frac{\partial P(a, t)}{\partial t}=\int w\left(a, a^{\prime}\right) d a^{\prime} P\left(a^{\prime} ; t\right)-\Gamma(a) P(a, t)$,

where the first term describes all transitions into state $a$ and, with

$$
\Gamma(a)=\int w\left(a^{\prime}, a\right) d a^{\prime},
$$

the second term describes all transitions out of state $a$. Equation (A7) can be written in the form

$$
\frac{\partial P}{\partial t}=-L P=-\int\left\langle a|L| a^{\prime}\right\rangle d a^{\prime} P\left(a^{\prime}, t\right),
$$

where

$$
\begin{aligned}
\left\langle a|L| a^{\prime}\right\rangle & \equiv-w\left(a, a^{\prime}\right)+\Gamma(a) \delta\left(a-a^{\prime}\right) \\
& =\lim _{\Delta t \rightarrow 0} \frac{P\left(a, t+\Delta t \mid a^{\prime} t\right)-\delta\left(a-a^{\prime}\right)}{\Delta t} .
\end{aligned}
$$

Equation (A3), with the help of (A5) and (A10), leads to the conditions

$$
\begin{aligned}
& w\left(\bar{a}_{0}, \bar{a}\right)=\frac{1}{P_{0}(a)} w\left(a, a_{0}\right) P_{0}\left(a_{0}\right), \\
& \Gamma(a)=\Gamma(\bar{a}) .
\end{aligned}
$$

These results are equivalent to the single condition

$$
K \tilde{L} K^{-1}=(1 / W) L W,
$$

where $W$ is an operator whose eigenvalue on a 
state of definite $a$ is $P_{0}(a)$, so that

$\left\langle a|(1 / W) L W| a_{0}\right\rangle=\left[1 / P_{0}(a)\right]\left\langle a|L| a_{0}\right\rangle P_{0}\left(a_{0}\right)$.

This result, which follows from the definition of the operator $L$ in terms of the conditional probability, is thus applicable to the master-equation form of the transport equation, as well as to the Fokker-Planck form when $L$ is approximated by a second-order differential operator. In either case, the complex conjugate of the adjoint equation

$$
L^{\dagger} \varphi_{n}=\Lambda_{n}^{*} \varphi_{n}
$$

when multiplied by $W$ and $K$, leads to

$$
W K \tilde{L} \varphi_{n}^{*}=W K \Lambda_{n} \varphi_{n}^{*}
$$

or

$$
W\left(K \tilde{L} K^{-1}\right) K \varphi_{n}^{*}=\Lambda_{n}\left(W K \varphi_{n}^{*}\right) .
$$

The use of (A13) leads to the result

$$
L\left(W K \varphi_{n}^{*}\right)=\Lambda_{n}\left(W K \varphi_{n}^{*}\right),
$$

so that $W K \varphi_{n}^{*}$ is an eigenfunction of $L$ with eigenvalue $\Lambda_{n}$ which can be identified with $P_{n}$. Thus,

$$
\varphi_{n}^{*}(a)=W^{-1} K P_{n}(a)=P_{n}(\bar{a}) / P_{0}(a) \text {. }
$$

To obtain $\hat{\varphi}_{n}^{*}$ we must replace $L$ by $L+\lambda \rho$ as in Eq. (5.10). If $\rho(a)=\rho(\bar{a})$, then Eq. (A13) with $L$ replaced by $L+\lambda \rho$ continues to be obeyed with the same $W$. Thus,

$$
\hat{\varphi}_{n}^{*}(a)=\hat{P}_{n}(\bar{a}) / P_{0}(a),
$$

and the Green's-function solution (5.16) can be written

$$
\hat{P}\left(a, t \mid a_{0} t_{0}\right)=\sum_{n} e^{-\hat{\Lambda}_{n}\left(t-t_{0}\right)} \frac{\hat{P}_{n}(a) \hat{P}_{n}\left(\bar{a}_{0}\right)}{P_{0}\left(a_{0}\right)} .
$$

Note that although $\hat{P}_{n}(a)$ is, in general, complex, $\hat{P}_{n}^{*}$ does not appear in Eq. (A21). For the Van der Pol oscillator, the complex variable $\beta=r e^{-i \varphi}$, where $\bar{r}=r, \bar{\varphi}=-\varphi$. The eigenvector $P_{n}$ requires a double index $n, \lambda$ and can be written

$$
\hat{P}_{n}=\hat{R}_{n}(r, \lambda) e^{i \lambda \varphi},
$$

so that

$$
\begin{aligned}
\hat{P}(r, \varphi, t & \left.; r_{0}, \varphi_{0}, 0\right) \\
& =\sum_{n, \lambda} \hat{R}_{n}(r, \lambda) \hat{R}_{n}\left(r_{0}, \lambda\right) e^{i \lambda\left(\varphi-\varphi_{0}\right)} e^{-\hat{\Lambda}_{\lambda, n} t},
\end{aligned}
$$

a result which we have previously given ${ }^{25}$ as valid even in the presence of detuning.

If one is concerned only with intensity fluctuations, one can restrict oneself to the $\lambda=0$ terms in (A23) and use the even variable $\rho=|\beta|^{2}=\gamma^{2}$ :

$$
\hat{P}\left(\rho, T ; \rho_{0}, 0\right)=\sum_{n} e^{-\hat{\Lambda}_{n} T} \hat{P}_{n}(\rho) \hat{P}_{n}\left(\rho_{0}\right) \text {. }
$$

\begin{tabular}{|c|c|c|c|c|c|c|}
\hline Field & $s$ & $p(m, T)$ & $n^{(2)}$ & $n^{(3)}$ & $n^{(4)}$ & $>n^{(4)}$ \\
\hline \multirow{6}{*}{$\begin{array}{l}\text { Laser } \\
\text { below } \\
\text { threshold } \\
\text { (thermal) } \\
\text { RWVP } \\
p<-10\end{array}$} & $<1$ & $\mathrm{FH}(65)$ & $\mathrm{FH}(65)$ & $\mathrm{FH}(65)^{\mathrm{a}}$ & $\mathrm{FH}(65)$ & \\
\hline & & & $\mathrm{SA}(65)$ & & & JOP(68) \\
\hline & $\sim 1$ & $\mathrm{FH}(65)$ & $\mathrm{FH}(65)$ & $\mathrm{FH}(65)$ & $\mathrm{FH}(65)$ & \\
\hline & & & & & & JOP(68) \\
\hline & & $\mathrm{FH}(65)$ & $\mathrm{FH}(65)$ & $\mathrm{FH}(65)$ & $\mathrm{FH}(65)$ & \\
\hline & $>1$ & JMP(65) & & & & \\
\hline \multirow{7}{*}{$\begin{array}{l}\text { Laser } \\
\text { near } \\
\text { threshold. } \\
\text { RWVP } \\
-10 \leq p \leq 10\end{array}$} & & & $\operatorname{DM}(67)$ & & & \\
\hline & & $\mathrm{SA}(66)$ & $\mathrm{SA}(66)$ & $\mathrm{A}(67)$ & & \\
\hline & $<1$ & $\operatorname{MDM}(70)$ & GGW(68) & CDKAH (67) & CDKAH (67) & GG(69) \\
\hline & & & & CKD(68) & CKD(68) & \\
\hline & $\sim 1$ & $\mathrm{P}(67)$ & $\mathrm{P}(67)$ & JOP(70) & JOP(70) & GG(69) \\
\hline & & $\operatorname{MDM}(70)$ & $\mathrm{CKD}(68)$ & CKD(68) & CKD(68) & \\
\hline & $>1$ & $\mathrm{JOP}(70)$ & JOP(70) & JOP(70) & JOP(70) & GG(69) \\
\hline \multirow{4}{*}{$\begin{array}{l}\text { Laser } \\
\text { above } \\
\text { threshold } \\
\text { RWVP } \\
p>10\end{array}$} & & $\mathrm{FH}(65)$ & $\mathrm{FH}(65)$ & $\mathrm{FH}(65)$ & $\mathrm{FH}(65)$ & \\
\hline & $<1$ & & $\mathrm{SA}(65)$ & & & \\
\hline & $\sim 1$ & $\mathrm{FH}(65)$ & FH (65) & $\mathrm{FH}(65)$ & $\mathrm{FH}(65)$ & \\
\hline & $>1$ & $\mathrm{FH}(65)$ & $\mathrm{FH}(65)$ & $\mathrm{FH}(65)$ & $\mathrm{FH}(65)$ & JOP(68) \\
\hline
\end{tabular}

Alternatively, the $\lambda \neq 0$ terms in Eq. (A22) disappear after the integration over $\varphi$ in Eq. (5.18).

TABLE III. Experimental photocount statistics of laser and thermal fields, a bibliography of initial papers, after Pike (Ref. 26). The year of publication is given in parentheses. The following abbreviations are used: A, Arecchi (Ref. 21); CDKAH, Chang, Detenbeck, Korenman, Alley, Jr., and Hochuly (Ref. 22); CKD, Chang, Korenman, and Detenbeck (Ref. 22); FH, Freed and Haus (Ref. 19); GG, Grace and Gamo (Ref. 80); GGW, Gamo, Grace, and Walter (Ref. 23); JMP, Johnson, McLean, and Pike (Ref. 81); JOP, Jakeman, Oliver, and Pike (Ref. 82); P, Pike (Ref. 83); MDM, Meltzer, Davis, and Mandel (Ref. 39); SA, Smith and Armstrong (Ref. 20). 


\section{APPENDIX B: DERIVATIVES OF $\hat{\Lambda}_{n}$ AND $\hat{P}_{n}$ WITH RESPECT TO $\lambda$}

Let $\hat{P}_{n}$ and $\hat{\Lambda}_{n}$ be defined by Eqs. (5.14) and (5.15) having the property of Eq. (5.17). Furthermore, let the derivatives of $\hat{P}_{n}$ with respect to $\lambda$ be defined by Eqs. (6.3). Differentiating the first of Eqs. (5.14), multiplying from the left-hand side by $\hat{\varphi}_{n}^{*}$ and integrating, one obtains with the help of Eqs. (6.3a) and (5.15),

$\hat{\Lambda}_{n}^{\prime}=\left(\hat{\varphi}_{n},\left\{\frac{\partial}{\partial \lambda}[L+\lambda \rho]\right\} \hat{P}_{n}\right)=\left(\hat{\varphi}_{n}, \rho \hat{P}_{n}\right)=\hat{\rho}_{n n}$,

a generalization of the well-known Feynman-Hell$\operatorname{man}^{79}$ theorem to the non-Hermitian case.

By differentiating Eq. (B1a) and using Eqs. (5.17) and (6.3a),

$$
\begin{aligned}
\hat{\Lambda}_{n}^{\prime \prime} & =\left(\hat{\varphi}_{n}^{\prime}, \rho \hat{P}_{n}\right)+\left(\hat{\varphi}_{n}, \rho \hat{P}_{n}^{\prime}\right) \\
& =\sum_{m} D_{m n}\left(\hat{\varphi}_{m}, \rho \hat{P}_{n}\right)+\sum_{m} D_{m n}\left(\hat{\varphi}_{n}, \rho \hat{P}_{m}\right) \\
& =\sum_{m} D_{m n}\left(\hat{\rho}_{m n}+\hat{\rho}_{n m}\right)=2 \sum_{m} \hat{\rho}_{n m} D_{m n},
\end{aligned}
$$

where the last step follows from the symmetry of $\rho$. Similarly one obtains,

$$
\begin{aligned}
& \hat{\Lambda}_{n}^{\prime \prime \prime}=2\left(\sum_{m} \hat{\rho}_{n m} E_{m n}+\sum_{l, m} \hat{\rho}_{l m} D_{m n} D_{l n}\right), \\
& \hat{\Lambda}_{n}^{\prime \prime \prime}=2\left(\sum_{m} \hat{\rho}_{n m} F_{m n}+3 \sum_{l, m} \hat{\rho}_{l m} E_{m n} D_{l m}\right) .
\end{aligned}
$$

With the use of (5.20), Eq. (B1a) can be written

$$
\hat{\rho}_{m n}=\sum_{i j} \rho_{i j} C_{j n} C_{i m},
$$

with $\rho_{i j}$ defined by Eq. (5.22), and the $C_{j n}$ determined by the secular equations (5.21). The coefficients $D_{m n}, E_{m n}, F_{m n}$, and $G_{m n}$ are calculated as follows. Differentiating the first of Eqs. (5.14), we find

$$
(L+\lambda \rho) \hat{P}_{n}^{\prime}+\rho \hat{P}_{n}=\hat{\Lambda}_{n}^{\prime} \hat{P}_{n}+\hat{\Lambda}_{n} \hat{P}_{n}^{\prime},
$$

but

$$
\rho \hat{P}_{n}=\sum_{m} \hat{P}_{m} \hat{\rho}_{m n}
$$

since

$$
\left(\hat{\varphi}_{l}, \rho \hat{P}_{n}\right)=\sum_{m}\left(\hat{\varphi}_{l}, \hat{P}_{m}\right) \hat{\rho}_{m n}=\hat{\rho}_{l n} .
$$

By inserting $\hat{P}_{n}^{\prime}$ from Eq. (6.3) and using (B5) and (5.14), Eq. (B4) becomes

$$
\sum_{m}\left(D_{m n} \hat{\Lambda}_{m}+\hat{\rho}_{m n}\right) \hat{P}_{m}=\sum_{m}\left(\hat{\Lambda}_{m}^{\prime} \delta_{n m}+D_{m n} \hat{\Lambda}_{n}\right) \hat{P}_{m},
$$

*Also at Physics Dept., City College of City University of New York, New York, N. Y. 10031.

Paper describes a portion of $\mathrm{Ph}$. D. thesis submitted to the Universidade Federal do Rio Grande do Sul, Pôrto Alegre, Brazil. Work done with partial support from Conselho Nacional de Pesquisas, Brazil. from which we conclude that

$$
\left(\hat{\Lambda}_{n}-\hat{\Lambda}_{m}\right) D_{m n}=\hat{\rho}_{m n}-\hat{\Lambda}_{m}^{\prime} \delta_{n m}
$$

or

$$
D_{m n}=\hat{\rho}_{m n} /\left(\hat{\Lambda}_{n}-\hat{\Lambda}_{m}\right), \quad m \neq n .
$$

Similarly by differentiating Eq. (5.14) successively, and by using Eqs. (6.3), one obtains

$$
\begin{aligned}
& E_{m n}=\frac{2}{\hat{\Lambda}_{n}-\hat{\Lambda}_{m}}\left(\sum_{l} \hat{\rho}_{m l} D_{l n}-\hat{\Lambda}_{n}^{\prime} D_{m n}\right), \quad m \neq n \quad \text { (B8b) } \\
& F_{m n}=\frac{3}{\hat{\Lambda}_{n}-\hat{\Lambda}_{m}}\left(\sum_{l} \hat{\rho}_{m l} E_{l n}-\hat{\Lambda}_{n}^{\prime} E_{m n}-\hat{\Lambda}_{n}^{\prime \prime} D_{m n}\right), \quad m \neq n \\
& G_{m n}=\frac{4}{\hat{\Lambda}_{n}-\hat{\Lambda}_{m}}\left(\sum_{l} \hat{\rho}_{m l} F_{l n}-\hat{\Lambda}_{n}^{\prime} F_{m n}-\frac{3}{2} \hat{\Lambda}_{n}^{\prime \prime} E_{m n}-\hat{\Lambda}_{m}^{\prime \prime \prime} D_{m n}\right), \\
& m \neq n . \quad \text { (B8d) }
\end{aligned}
$$

The diagonal coefficients are calculated from Eq. (5.15) in which we expand $\hat{\varphi}_{n}$ and $\hat{P}_{n}$ in a Taylor series and collect terms of same order:

$$
\begin{aligned}
1= & \left(\hat{\varphi}_{n}(\lambda+\Delta), \hat{P}_{n}(\lambda+\Delta)\right) \\
= & \left(\hat{\varphi}_{n}, \hat{P}_{n}\right)+\Delta\left[\left(\hat{\varphi}_{n}, \hat{P}_{n}^{\prime}\right)+\left(\hat{\varphi}_{n}^{\prime}, \hat{P}_{n}\right)\right] \\
& +\frac{1}{2} \Delta^{2}\left[\left(\hat{\varphi}_{n}, \hat{P}_{n}^{\prime \prime}\right)+2\left(\hat{\varphi}_{n}^{\prime}, \hat{P}_{n}^{\prime}\right)+\left(\hat{\varphi}_{n}^{\prime \prime}, \hat{P}_{n}\right)\right]+\cdots .
\end{aligned}
$$

Thus, all coefficients of the powers of $\Delta$ must be zero. This implies, for example,

$$
\begin{aligned}
0 & =\left(\hat{\varphi}_{n}, \hat{P}_{n}^{\prime}\right)+\left(\hat{\varphi}_{n}^{\prime}, \hat{P}_{n}\right) \\
& =\sum_{m} D_{m n}\left(\hat{\varphi}_{n}, \hat{P}_{m}\right)+\sum_{m} D_{m n}\left(\hat{\varphi}_{m}, \hat{P}_{n}\right)=2 D_{n n} .
\end{aligned}
$$

Thus,

$$
D_{n n}=0 \text { for all } n,
$$

and similarly,

$$
\begin{aligned}
& E_{n n}=-\sum_{m} D_{m n}^{2}, \\
& F_{n n}=-\sum_{m} D_{m n} E_{m n}, \\
& G_{n n}=-4 \sum_{m} D_{m n} F_{m n}-3 \sum_{m} E_{m n}^{2} .
\end{aligned}
$$

The eigenvalue derivatives $\Lambda_{n}^{\prime}, \Lambda_{n}^{\prime \prime}, \Lambda_{n}^{\prime \prime \prime}, \Lambda_{n}^{\prime \prime \prime \prime}$ and wave-function expansion coefficients $D_{m n}, E_{m n}$, $F_{m n}, G_{m n}$ are used in Sec. VI to calculate the exact moments $\left\langle\omega^{r}\right\rangle$, i.e., the exact factorial moments $n^{(r)}(T)$ [Eq. (2.10) of the photocount distribution].

${ }^{\ddagger}$ Present address: Universidade Federal do Rio Grande do Sul, Departmento de Fisica, Pôrto Alegre, Brazil.

${ }^{1}$ D. Slepian, Bell Syst. Tech. J. 37, 163 (1958); U. Grenander, H. O. Pollak, and D. Slepian, J. Soc. Ind. Appl. Math. 7, 374 (1959).

${ }^{2}$ E. Jakeman and E. R. Pike, J. Phys. A 2, 115 (1969). 
${ }^{3}$ G. Bédard, Phys. Rev. 151, 1038 (1966)

${ }^{4}$ R. Graham and H. Haken, Z. Phys. 237, 31 (1970).

${ }^{5}$ V. DeGiorgio and M. O. Scully, Phys. Rev. A 2, 1170 (1970).

${ }^{6}$ M. Lax, Phys. Rev. 145, 110 (1966).

${ }^{7}$ The moments of all (field and atom) noncommuting noise sources were derived by a single generalized Einstein relation [Eq. (2.9) of Ref. 6]. In connection with an application to the derivation of the phase linewidth (Ref. 8), this Einstein relation and the resulting moments (Ref. 6) were presented at the Puerto Rico Conference on the Physics of Quantum Electronics, 1965. The atomic moments were explicitly obtained as atomic operators with the help of an atomic-operator algebra introduced much earlier (Ref. 9). Up to that time, the operator dependence of the atomic noise sources had not been recognized. For example, the paper presented by H. Haken, H. Risken, H. Sauerman, and W. Weidlich (unpublished) made use of noncommuting atomic noise sources introduced by Sauerman (Ref. 10), whose moments did not have the correct operator dependence. This defect was then remedied by Haken and Weidlich (Ref. 11) making use of our operator algebra (Ref. 9) but not of the Einstein relation. This procedure was not (as the Einstein relation is) capable of directly yielding the field (harmonic oscillator) noise sources introduced by Senitzky (Ref. 13) which differ slightly from ours. It is possible to treat the harmonic oscillator directly (Ref. 6) with the help of the Einstein relation or as a special multilevel atom with regular spacing with noise sources that agree with each other in the two cases and disagree slightly with the Senitzky noise sources.

${ }^{8}$ M. Lax, in Physics of Quantum Electronics, edited by P. L. Kelley, B. Lax, and P. E. Tannenwald (McGraw-Hill, New York, 1966).

${ }^{9}$ M. Lax, Phys. Rev. 129, 2341 (1963).

${ }^{10}$ H. Sauerman, Z. Phys. 188, 480 (1965); Z. Phys. 189, 312 (1966).

${ }^{11}$ H. Haken and W. Weidlich, Z. Phys. 189, 1 (1966).

${ }^{12}$ H. Haken, Z. Phys. 190, 327 (1966).

${ }^{13}$ I. R. Senitzky, Phys. Rev. 119, 670 (1960); Phys. Rev. 124, 642 (1961).

${ }^{14}$ M. Lax and W. H. Louisell, IEEE J. Quantum Electron. QE-3, 47 (1967). This paper was presented at the Phoenix International Conference on Quantum Electronics, 1966.

${ }^{15}$ R. D. Hempstead and M. Lax, Phys. Rev. 161, 360 (1967), Appendix A. This work was presented by R. D. Hempstead to the Dept. of Electrical Engineering of Massachusetts Institute of Technology in 1965, in partial fulfillment of the requirements for a Master of Science Degree.

${ }^{16}$ The intensity relaxation rate is $\langle\Lambda\rangle / T$, where the dimensionless $\langle\Lambda\rangle$ is tabulated in Table I and has the value 5.853 at threshold. The scaling time $T$ (see Refs. 17 and 56 for an exact definition of the scaling time $T$ and other Van der Pol parameters) can be written $T=\left(2 n^{\text {th }}\right) /(1.128 \gamma)$, where 1.128 is the threshold value of $\langle\rho\rangle$ and $\gamma$ is the cavity decay rate $\left(\sim 10^{7} \%\right.$ sec). Thus, the typical time $(1 / \gamma)$ is scaled up by the large factor $n^{\mathrm{th}} \approx 10^{4}$.

${ }^{17}$ M. Lax, Phys. Rev. 157, 213 (1967), Fig. 1.

${ }^{18}$ M. Lax, Phys. Rev. 160, 290 (1967), Ref. 10.

${ }^{19} \mathrm{C}$. Freed and H. A. Haus, in Physics of Quantum Electronics, edited by P. L. Kelley et al. (McGraw-Hill, New York, 1966), p. 715 .

${ }^{20}$ A. W. Smith and J. A. Armstrong, in Physics of Quantum Electronics, edited by P. L. Kelley et al. (McGraw-Hill, New York, 1966), p. 701; Phys. Rev. Lett. 16, 1169 (1966).

${ }^{21}$ F. T. Arecchi, in Quantum Optics, edited by R. J. Glauber (Academic, New York, 1969), p. 57.

${ }^{22}$ R. F. Chang, R. W. Detenbeck, V. Korenman, C. O. Alley, Jr., and U. Hochuli, Phys. Lett. A 25, 272 (1967); R. F. Chang, V. Korenman, and R. W. Detenbeck, Phys. Lett. A 26, 417 (1968).

${ }^{23}$ H. Gamo, R. E. Grace, and T. J. Walter, IEEE J. Quantum Electron. QE -4, 344 (1968). Measurements of the second factorial moment $n^{(2)}$ and of the linewidth of laser intensity fluctuations were first reported to the Rochester Conference on Coherence and Quantum Optics, 1966.

${ }^{24}$ F. Davidson and L. Mandel, Phys. Lett. A 25, 700 (1967).

${ }^{25}$ M. Lax, in Statistical Physics, Phase Transitions and Superfluidity, edited by M. Chrétien, E. P. Gross, and S. Deser (Gordon and Breach, New York, 1968), Vol. 2.

${ }^{26} \mathrm{E}$. R. Pike (private communication).

${ }^{27}$ The first applications of this quantum-classical correspondence to phase and intensity fluctuations were made in Ref. 14. The first extension from two-time to many-time operators was made in Chap. 11 of the 1966 Brandeis Lectures (Ref. 25). An extension to many operators (including atomic as well as field operators) and many times was made in Ref. 28. At the same time, Haken and Weidlich (Ref. 29) introduced a modified-density-operator technique capable in principle of yielding multitime field-operator averages (in the presence of atoms), and Haake (Ref. 30) has recently extended this technique to the non-Markoffian case. The techniques of Refs. 29 and 30 did not, however, provide the classical technique of taking multitime averages involving atomic as well as field operators introduced in Ref. 28. Applications of a correspondence technique to two-level lasers was made by Haken, Risken, and Weidlich (Ref. 31), and applications to threelevel lasers was made by Gordon (Ref. 32) and by Lax and Yuen (Ref. 33).

${ }^{28}$ M. Lax, Phys. Rev. 172, 350 (1968).

${ }^{29}$ H. Haken and W. Weidlich, Z. Phys. 205, 96 (1967).

${ }^{30}$ F. Haake, Phys. Rev. A 3, 1723 (1971).

${ }^{31}$ H. Haken, H. Risken, and W. Weidlich, Z. Phys. 206, 355 (1967).

${ }^{32}$ J. P. Gordon, Phys. Rev. 161, 367 (1967)

${ }^{33}$ M. Lax and H. Yuen, Phys. Rev. 172, 362 (1968).

${ }^{34}$ H. Risken and H. D. Vollmer, Z. Phys. 201, 323 (1967); H. Risken, Z. Phys. 191, 302 (1966); S. Grossman and P. H. Richter, Z. Phys. 242, 458 (1971).

${ }^{35}$ F. T. Arecchi, G. S. Rodari, and A. Sona, Phys. Lett. A 25, 59. (1967); F. T. Arecchi, M. Giglio, and A. Sona, Phys. Lett. A 25, 341 (1967).

${ }^{36}$ E. D. Hinckley and C. Freed, Phys. Rev. Lett. 23, 277 (1969); K. R. Manes and A. Siegman, Phys. Rev. Lett. 4, 373 (1971); H. Gerhardt, H. Welling, and A. Güttner, Z. Physik (to be published).

${ }^{37}$ T. S. Jaseja, A. Javan, and C. H. Townes, Phys. Rev. Lett. 10, 165 (1963); A. E. Siegman, B. Daino, and K. R. Manes, IEEE J. Quantum Electron. QE -3, 160 (1967); Yu. N. Zaitsev and D. P. Stepanoff, Zh. Eksp. Teor. Fiz. Pis'ma Red. 6, 733 (1967) [JETP Lett. 6, 209 (1967)]; A. E. Siegman and R. Arrathoon, Phys. Rev. Lett. 20, 901 (1968); Yu. P. Egorov, Zh. Eksp. Teor. Fiz. Pis'ma Red. 8, 525 (1968) [JETP Let+. 8, 320 (1969)]; J. A. Armstrong and Archibald W. Smith, Appl. Phys. Lett. 4, 196 (1964); S. W. Crowe and R. M. Craig, Appl. Phys. Lett. 5, 72 (1964); W. E. Ahearn and J. W. Growe, IEEE J. Quantum Electron. QE-2, 597 (1966).

${ }^{38}$ M. Lax and M. Zwanziger, Phys. Rev. Lett. 24, 937 (1970).

${ }^{39}$ D. Meltzer, W. Davis, and L. Mandel, Appl. Phys. Lett. 17, 242 (1970).

${ }^{40}$ E. Jakeman, C. J. Oliver, E. R. Pike, M. Lax, and M. Zwanziger, J. Phys. A 3, L52 (1970).

${ }^{41}$ R. J. Glauber, Phys. Rev. Lett. 10, 84 (1963); in Quantum Optics and Electronics, edited by C. DeWitt et al. (Gordon and Breach, New York, 1965).

${ }^{42}$ R. J. Glauber, in Quantum Optics, edited by R. J. Glauber (Academic, New York, 1969), p. 15. In this paper the detector is replaced by a harmonic oscillator and then treated exactly rather than with the help of perturbation theory. This field, however, is still treated as free.

${ }^{43}$ L. Mandel and E. Wolf, Rev. Mod. Phys. 37, 231 (1965).

${ }^{44}$ J. R. Klauder and E. C. G. Sudarshan, Fundamentals of Quantum Optics (Benjamin, New York, 1968), Chap. 8.

${ }^{45}$ P. L. Kelley and W. H. Kleiner, Phys. Rev. 136, A316 
(1964).

${ }^{46}$ B. R. Mollow, Phys. Rev. 168, 1896 (1968).

${ }^{47}$ Details of derivations omitted here, and extensive tables of input data and results are available from M. Lax in a previous draft of this paper.

${ }^{48}$ F. A. Johnson, R. Jones, T. P. McLean, and E. R. Pike, Phys. Rev. Lett. 16, 589 (1966); I. de Lotto, P. F. Manfredi, and P. Principio, Energ. Nucl. (Milan) 11, 557 (1964).

${ }^{49}$ The intensity $\rho(T)$ is more conventionally called $I(T)$, see for example, Ref. 43. In our laser example, $\rho(T)$ will represent a dimensionless scaled intensity. Because of the presence of the efficiency factor $\epsilon$, either scaled or unscaled intensities can be used with impunity since quantities to be compared with experiment will be expressed in a form independent of $\epsilon$.

${ }^{50}$ L. Mandel, Proc. Phys. Soc. Lond. 72, 1037 (1958). See also L. Mandel, in Progress in Optics, edited by E. Wolf (North-Holland, Amsterdam, 1963), Vol. 2, p. 242.

${ }^{51}$ M. O. Scully and W. E. Lamb, Jr., Phys. Rev. 179, 368 (1969).

${ }^{52}$ W. H. Louisell, Radiation and Noise in Quantum Electronics (McGraw-Hill, New York, 1964), p. 104; W. H. Louisell and L. R. Walker, Phys. Rev. 137, B204 (1965); R. Kubo, J. Phys. Soc. Jap. 17, 1100 (1962); F. Coester and H. Kümmel, Nucl. Phys. 17, 477 (1960).

${ }^{53}$ R. J. Glauber, Phys. Rev. Lett. 10, 84 (1963); Phys. Rev. 130, 2529 (1963); Phys. Rev. 131, 2766 (1963).

${ }^{54}$ E. C. G. Sudarshan, Phys. Rev. Lett. 10, 277 (1963);

Proceedings of the Symposium on Optical Lasers (Wiley, New York, 1963), p. 43; C. L. Mehta and E. C. G. Sudarshan, Phys. Rev. 138, B274 (1965). For a review on coherent states, see also L. Mandel and E. Wolf, Rev. Mod. Phys. 37, 231 (1965)

${ }^{55} \mathrm{H}$. Haken and W. Weidlich, in Quantum Optics, edited by R. J. Glauber (Academic, New York, 1969); M. Scully, in Quantum Optics, edited by R. J. Glauber (Academic, New York, 1969); M. Lax, in Statistical Physics, Phase Transition and Superfluidity, edited by H. Chretian. et al. (Gordon and Breach, New York, 1968). For a recent review, see H. Haken, in Handbuch der Physik, edited by S. Flügge (Springer, Berlin, 1970), Vol. XXV/2c.

${ }^{56}$ M. Lax and W. H. Louisell, Phys. Rev. 185, 568 (1969) (especially Sec. 12).

${ }^{57}$ M. Lax, Rev. Mod. Phys. 38, 541 (1966) [see especially Eqs. (3.27)-(3.28)] .

${ }^{58}$ M. Lax, Rev. Mod. Phys. 38, 359 (1966) [see Eqs. (5.13) and (7B.1)].

${ }^{59}$ M. Kac, Trans. Am. Math. Soc. 59, 401 (1946); Berkeley Symposium on Mathematics, Statistics and Probability (University of California Press, Berkeley, Calif., 1951), p. 189; A. J. F. Siegert, IRE Trans. Inf. Theory 3, 38 (1957); IRE Trans. Inf. Theory 4, 4 (1958).

${ }^{60}$ Reference 58, Eqs. (4.2) and (4.8).

${ }^{61}$ P. M. Morse and H. Feshbach, Methods of Theoretical Physics (McGraw-Hill, New York, 1953), p. 884.

${ }^{62}$ Reference 61, p. 864.

${ }^{63}$ R. D. Hempstead and M. Lax (unpublished).

${ }^{64} \mathrm{P}$. M. Morse and H. Feshbach, Ref. 61, p. $437 \mathrm{ff}$.

${ }^{65}$ R. E. Bellman, R. E. Kalaba, and J. A. Lockett, Numerical Inversion of the Laplace Transform (American Elsevier, New York,
1966).

${ }^{66}$ V. I. Krylov and N. S. Skoblya, Handbook of Numerical Inversion of Laplace Transforms (Israel Program for Scientific Translations, Jerusalem, 1969).

${ }^{67}$ F. B. Hildebrand, Introduction to Numerical Analysis

(McGraw-Hill, New York, 1956), Chap. 8.

${ }^{68} \mathrm{~J}$. R. Westlake, A Handbook of Numerical Matrix Inversion (Wiley, New York, 1968), Chap. 4; W. Gautschi, Math. Comput. 23, 109 (1969).

${ }^{69}$ H. E. Salzer and R. Zucher, Bull. Am. Phys. Soc. 55, 1004 (1949).

${ }^{70}$ S. O. Rice, Bell Syst. Tech. J. 24, 46 (1945); L. Mandel, Proc. Phys. Soc. Lond. 74, 233 (1959).

${ }^{71}$ M. Lax, Rev. Mod. Phys. 32, 25 (1960).

${ }^{72}$ R. J. Glauber, in Physics of Quantum Electronics, edited by P. L. Kelley et al. (McGraw-Hill, New York, 1966), p. 808.

${ }^{73}$ G. Lachs, Phys. Rev. 138, B1012 (1965). See also H. Morawitz, Phys. Rev. 139, A1072 (1965), G. Lachs, J. Appl. Phys. 42, 602 (1971).

${ }^{74}$ E. Jakeman and E. R. Pike, J. Phys. A 2, 115 (1969).

${ }^{75}$ A. K. Jaiswal and C. L. Mehta, Phys. Rev. A 2, 168 (1970).

${ }^{76}$ E. Jakeman and E. R. Pike, J. Phys. A 1, 128 (1968).

${ }^{77}$ B. Numerov, Publ. Observatoire Central Astrophys. Russ. 2, 188 (1933).

${ }^{78}$ D. R. Hartree, The Calculation of Atomic Structures (Wiley, New York, 1957), pp. 71-85; Numerical Analysis (Clarendon, Oxford, England, 1958), pp. 142-162.

${ }^{79}$ R. P. Feynman, Phys. Rev. 56, 340 (1939); H. Hellmann, Einführung in die Quantenchemie (Franz Deuticke, Leipzig, 1937), p. 285.

${ }^{80}$ R. E. Grace and H. Gamo, J. Opt. Soc. Am. 59, 504 (1969).

${ }^{81}$ F. A. Johnson, T. P. McLean, and E. R. Pike, in Physics of Quantum Electronics, edited by P. L. Kelley et al. (McGraw-Hill, New York, 1966), p. 706

${ }^{82}$ E. Jakeman, C. J. Oliver, and E. R. Pike, J. Phys. A 1, 406

(1968); J. Phys. A 1, 497 (1968); J. Phys. A 3, L52 (1970).

${ }^{83}$ E. R. Pike, in Quantum Optics, edited by R. J. Glauber (Academic, New York, 1969), p. 160.

${ }^{84}$ C. Helstrom, Proc. Phys. Soc. Lond. 83, 777 (1964).

${ }^{85}$ A. K. Jaiswal and C. L. Mehta, Phys. Rev. A 2, 168 (1970).

${ }^{86}$ E. Jakeman and E. R. Pike, J. Phys. A 1, 128 (1968); J. Phys. A 1, 627 (1968); J. Phys. A 2, 115 (1969).

${ }^{87}$ L. Mandel, Proc. Phys. Soc. Lond. 72, 1037 (1958); Proc.

Phys. Soc. Lond. 74, 233 (1959); in Quantum Electronics, edited

by P. Grivet et al. (Columbia U.P., New York, 1964), p. 101

${ }^{88}$ T. P. McLean and E. R. Pike, Phys. Lett. 15, 318 (1965).

${ }^{89}$ J. Pěrina, Phys. Lett. A 24, 333 (1967).

${ }^{90}$ J. Pěrina, and R. Horák, J. Phys. A 2, 702 (1969).

${ }^{91}$ E. M. Purcell, Nature (Lond.) 178, 1449 (1956).

${ }^{92}$ H. Risken, Z. Phys. 251, 231 (1972).

${ }^{93}$ R. Graham and H. Haken, Z. Phys. 243, 289 (1971).

${ }^{94}$ S. R. De Groot and P. Mazur, Nonequilibrium

Thermodynamics (North-Holland, Amsterdam, 1962), Chap. VII.

${ }^{95} \mathrm{M}$. Lax, in Dynamical Processes in Solid State Optics, edited by R. Kubo and H. Kamimura (Benjamin, New York, 1967), pt. I, Eq. (22C1). 